\title{
EQUIVALENCE AND STRONG EQUIVALENCE OF ACTIONS ON HANDLEBODIES
}

\author{
JOHN KALLIONGIS AND ANDY MILLER
}

\begin{abstract}
An algebraic characterization is given for the equivalence and strong equivalence classes of finite group actions on 3-dimensional handlebodies. As one application it is shown that each handlebody whose genus is bigger than one admits only finitely many finite group actions up to equivalence. In another direction, the algebraic characterization is used as a basis for deriving an explicit combinatorial description of the equivalence and strong equivalence classes of the cyclic group actions of prime order on handlebodies with genus larger than one. This combinatorial description is used to give a complete closed-formula enumeration of the prime order cyclic group actions on such handlebodies.
\end{abstract}

The study of finite group actions on compact 2-manifolds has an intricate history which may be traced back well into the 19th century. (For background on this see the survey article and bibliography [E1].) Many of the results which have been established in this study may be obtained through the following algebraic setup: if $G$ is a finite group acting effectively on the compact surface $S$, then there is an associated group extension

$$
1 \rightarrow \Pi_{1}(S) \rightarrow E \rightarrow G \rightarrow 1
$$

obtained by lifting the action to the universal covering of $S$. Given such a group extension whose abstract kernel $G \rightarrow \operatorname{Out}\left(\Pi_{1}(S)\right)$ preserves the peripheral structure of $\Pi_{1}(S)$ (if $S$ is bounded), the affirmative solution to the Nielsen realization problem $[\mathbf{K}]$ implies that there is a $G$-action which corresponds to it. Furthermore it is known that two $G$-actions on $S$ associated with the same group extension (in the sense of equivalence of exact sequences) are equivalent-in fact even strongly equivalent. (In the closed case a proof may be found in $[\mathbf{Z Z}]$ - for the bounded case a similar approach works using $[\mathbf{M}]$.) Within this setting an enumeration of the equivalence classes of $G$-actions on a given surface is theoretically possible through the techniques employed in [S] and again in [E3]. The key idea is that the set of free $G$-actions with a fixed quotient surface are algebraically categorized by epimorphisms from the fundamental group of the quotient surface to $G$. Actions which are not free may be categorized in a similar way using the orbifold fundamental group of the quotient orbifold.

In this paper we will utilize a similar approach to study the equivalence and strong equivalence classes of finite group actions on 3-dimensional handlebodies. In

Received by the editors April 17, 1987.

1980 Mathematics Subject Classification (1985 Revision). Primary 57M99; Secondary 57S25, $57 \mathrm{M} 12,57 \mathrm{M} 15$.

Key words and phrases. Handlebody, finite group action, handlebody orbifold, graph of groups, equivalence and strong equivalence of actions. 
actuality the development differs considerably from its 2-dimensional counterpart. On one hand the realization problem sometimes has a negative solution in this setting - although a complete solution was given in [MMZ]. On the other hand we shall see that two actions corresponding to the same group extension need not be equivalent, let alone strongly equivalent. Despite these differences with the 2dimensional case, we will show that an enumeration of the equivalence and strong equivalence classes of $G$-actions on a given handlebody is theoretically possible, and we will describe this enumeration in an explicit combinatorial fashion when $G$ is cyclic of prime order. To give a randomly chosen illustration, our theory implies that there are exactly 87,108 equivalence classes of $\mathbf{Z}_{13}$-actions on the handlebody of genus 157 (see Corollary 2.4). The results we obtain are potentially useful in studying finite group actions on arbitrary compact 3-manifolds via their Heegaard decompositions into the sum of two handlebodies.

To describe the setting of our results more accurately we now fix the terminology. We will denote the orientable 3-dimensional handlebody of genus $g$ by $V_{g}$. The fundamental group of $V_{g}$ is denoted by $F_{g}$; it is free of rank $g$. A $G$-action on $V_{g}$ is a group monomorphism $\phi: G \rightarrow \mathrm{Homeo}^{+}\left(V_{g}\right)$ where $\mathrm{Homeo}^{+}\left(V_{g}\right)$ is the group of orientation-preserving PL homeomorphisms of $V_{g}$. (Note that our actions as defined are always effective and orientation-preserving.) The group $G$ will always be assumed to be finite. Two $G$-actions $\phi_{1}$ and $\phi_{2}$ on $V_{g}$ are said to be equivalent if and only if there is $h \in \operatorname{Homeo}^{+}\left(V_{g}\right)$ so that $\phi_{2}(x)=h \phi_{1}(x) h^{-1}$ for all $x \in G$ (alternatively we shall write $\phi_{2}=\mu(h) \phi_{1}$ ). If such a homeomorphism $h$ may be found which is homotopic to the identity map on $V_{g}$ then we say that $\phi_{1}$ and $\phi_{2}$ are strongly equivalent. Two actions $\phi_{1}$ and $\phi_{2}$ are said to have the same quotient type if and only if the orbifold quotients $V_{g} / \phi_{1}$ and $V_{g} / \phi_{2}$ are homeomorphic (in the orbifold sense). The actions $\phi_{1}$ and $\phi_{2}$ are homotopic provided that they produce the same abstract kernel $G \rightarrow \operatorname{Out}\left(F_{g}\right)$ when composed with the induced homomorphism $\Psi:$ Homeo $^{+}\left(V_{g}\right) \rightarrow \operatorname{Out}\left(F_{g}\right)$; that is $\Psi \phi_{1}=\Psi \phi_{2}$. When $g>1$, two actions are homotopic exactly when they correspond to the same group extensions as discussed in the first paragraph (since $F_{g}$ has trivial center). It is immediately apparent that the following relationships hold among the various concepts:

$$
\left.\left\{\begin{array}{c}
\text { strongly } \\
\text { equivalent }
\end{array}\right\} \Rightarrow\left\{\begin{array}{c}
\text { homotopic and } \\
\text { equivalent }
\end{array}\right\} \Rightarrow \text { homotopic }\right\}
$$

Note that two strongly equivalent actions are always homotopic and they can be seen to correspond to the same group extension; thus the strong equivalence classes give a measure of the number of different ways in which a fixed group extension can be realized by an action. As remarked in the first paragraph, each of the implications indicated above is reversible for finite group actions on compact surfaces. On the other hand, for $\mathbf{Z}_{p}$-actions on handlebodies ( $p$ prime) we will show that both of the implications are reversible only when $p=2$ (see Corollary 4.4). We also show that each of these implications is reversible for free $\mathbf{Z}_{p}$-actions on handlebodies. By different methods it was shown in $[\mathbf{P}]$ that free (not necessarily prime) cyclic actions are always equivalent when $g>1$.

For a fixed finite group $G$ let $\mathscr{E}\left(G, V_{g}\right)$ denote the set of all equivalence classes of $G$-actions on $V_{g}$. In $\S 1$ of this paper we will give an algebraic characterization of $\mathscr{E}\left(G, V_{g}\right)$. This is obtained by considering handlebody coverings over the possible 
3-orbifolds which can arise as quotients of these actions. The Equivariant Dehn Lemma and Thurston's generalization of the Smith Conjecture imply that these orbifold quotients are handlebody orbifolds as described in $[\mathbf{M M Z}]$ - that is they are homeomorphic to $V(\Gamma, \mathbf{G})$ where $(\Gamma, \mathbf{G})$ is a $G$-admissible graph of groups in canonical form (see Proposition 1.1). One direct application of the characterization which we obtain in $\S 1$ is that each handlebody of genus larger than one admits only finitely many finite group actions up to equivalence (Corollary 1.4). Of course this is not true for handlebodies of genus 0 or 1 . In $\S 2$, the algebraic characterization of $\mathscr{E}\left(G, V_{g}\right)$ is used to give a combinatorial description of the sets $\mathscr{E}\left(\mathbf{Z}_{p}, V_{g}\right)$ ( $p$ prime, $g>1$ ). In the third section we describe an algebraic classification of the collection $\mathscr{S} \mathscr{E}(C)$ of strong equivalence classes of $G$-actions in $C$, where $C$ is the intersection of a homotopy class of actions with a quotient-type class of actions. Frequently - for instance when $G$ has prime order- $C$ equals the entire homotopy class in which it is contained, but this is not always the case as illustrated in $\S 4$. The results of $\S 3$ are also used in $\S 4$ to give a complete combinatorial description of the set $\mathscr{S} \mathscr{E}(C)$ of strong equivalence classes of $\mathbf{Z}_{p}$-actions on $V_{g}(g>1)$ in a fixed homotopy class $C$. It should be noted that the enumerations of $\mathbf{Z}_{p}$-actions on $V_{g}$ obtained in $\S \S 2$ and 4 do not apply when $g=1$ (so that $V_{g}$ is a solid torus). This is primarily due to the fact that $\operatorname{Cent}\left(F_{g}\right)$ is nontrivial and that a key lemma (Lemma 2.2) fails in this case. In a subsequent paper, we will apply the theorems of $\S \S 1$ and 3 to completely describe all finite group actions on the solid torus up to equivalence, strong equivalence and homotopy.

TABLE

\begin{tabular}{|c|c|c|c|c|c|c|c|c|}
\hline $\begin{array}{l}\text { quotient type } \\
\text { of } C:(s, t)\end{array}$ & $(0,0)$ & $(1,0)$ & $(2,0)$ & $(3,0)$ & $(4,0)$ & $(5,0)$ & $(0,5)$ & $(1,5)$ \\
\hline $\begin{array}{l}\text { \# equivalence } \\
\text { classes in } C\end{array}$ & 1 & 2 & 3 & 4 & 5 & 6 & 6 & 12 \\
\hline $\begin{array}{l}\text { \# strong equiv. } \\
\text { classes in } C\end{array}$ & 1 & 4 & 16 & 64 & 256 & 1024 & 32 & 128 \\
\hline $\begin{array}{l}\text { \# strong equiv. } \\
\text { classes in each } \\
\text { equiv. class of } C\end{array}$ & 1 & $2(2)$ & $\begin{array}{l}4(2) \\
8(1)\end{array}$ & $\begin{array}{c}8(2) \\
24(2)\end{array}$ & $\begin{array}{l}16(2) \\
64(2) \\
96(1)\end{array}$ & $\begin{array}{c}32(2) \\
160(2) \\
320(2)\end{array}$ & $\begin{array}{c}1(2) \\
5(2) \\
10(2)\end{array}$ & $\begin{array}{l}2(4) \\
10(4) \\
20(4)\end{array}$ \\
\hline
\end{tabular}

Taken altogether the results described above give a complete picture of the prime order cyclic group actions on handlebodies with genus larger than 1. For a fixed $g$

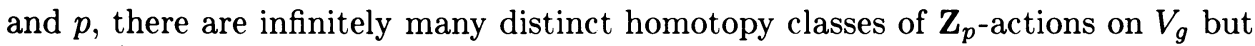
$\mathrm{Homeo}^{+}\left(V_{g}\right)$ acts by conjugation on these classes preserving equivalence and strong equivalence of actions. Thus two homotopy classes in the same orbit under the action of $\mathrm{Homeo}^{+}\left(V_{g}\right)$ have the same number of equivalence and strong equivalence classes. Moreover, two homotopy classes are in the same orbit if and only if they have the same quotient type (Proposition 2.6). The quotient types in turn depend only on the topological type of the fixed point set of the actions; thus the different quotient types of homotopy classes are determined by the number $s$ of circle components together with the number $t$ of arc components in the fixed point set. In particular, the quotient of the set of homotopy classes of $\mathbf{Z}_{p}$-actions on $V_{g}$ under the action of $\mathrm{Homeo}^{+}\left(V_{g}\right)$ is finite. To illustrate these ideas, consider the set of $\mathbf{Z}_{5}$-actions on $V_{21}$. By Corollary 2.4, there are 8 quotient types and 39 equivalence classes of such actions. Using information from this corollary, from Theorem 4.2 
and from Corollary 4.3 we obtain the information describing any homotopy class $C$ of $\mathbf{Z}_{5}$-actions on $V_{21}$ (see Table).

1. Equivalence of actions of arbitrary groups. In this section we give an algebraic characterization of the set $\mathscr{E}\left(G, V_{g}\right)$ of equivalence classes of actions of the finite group $G$ on the handlebody $V_{g}$. Of central importance in deriving this characterization is the identification of the quotient of any such action as an orbifold handlebody (Proposition 1.1). As described in [MMZ, §3], an (orientable) orbifold handlebody is formed by gluing together orbifold 0-handles (orientable 3-orbifolds covered by $B^{3}$ ) and orbifold 1-handles (products with $I$ of orientable 2-orbifolds covered by $\left.B^{2}\right)$ using a core graph of groups $(\Gamma, \mathbf{G})$ which satisfies a set of normalized conditions (described below). The handlebody orbifold with core $(\Gamma, \mathbf{G})$ is denoted by $V(\Gamma, \mathbf{G})$. It has fundamental group $\Pi_{1}^{\text {orb }}(V(\Gamma, \mathbf{G})) \simeq \Pi_{1}(\Gamma, \mathbf{G})$ and Euler characteristic $\chi^{\text {orb }}(V(\Gamma, \mathbf{G}))=\chi(\Gamma, \mathbf{G})$.

For completeness we will describe the normalized conditions on a graph of groups $(\Gamma, \mathbf{G})$. If $e$ is an (oriented) edge of $\Gamma$ then $\bar{e}$ is the oppositely oriented edge, and the initial and terminal vertices of $e$ are denoted by $\delta_{0}(e)$ and $\delta_{0}(\bar{e})$ respectively. The edge group of $\mathbf{G}$ corresponding to the edge $e$ is $G_{e}$ and the vertex group corresponding to the vertex $u$ is $G_{\iota}$. For each edge $e$ there is an edge-to-vertex monomorphism $f_{e}: G_{e} \rightarrow G_{\omega}$. The normalized conditions on a finite graph of groups $(\Gamma, \mathbf{G})$ consist of (N0)-(N4) as follows:

(N0) If $e$ and $e^{\prime}$ are edges in $\Gamma$ with $\delta_{0}(e)=\delta_{0}\left(e^{\prime}\right)$ and image $\left(f_{e}\right)$ is conjugate into image $\left(f_{e^{\prime}}\right)$, then image $\left(f_{e}\right) \leq \operatorname{image}\left(f_{e^{\prime}}\right)$.

(N1) Each vertex group $G_{o}$ is isomorphic to a finite subgroup of $S O(3)$ (thus it is cyclic, dihedral, tetrahedral, octahedral, or icosahedral).

(N2) If $e$ is an edge of $\Gamma$ and $G_{e} \neq 1$, then image $\left(f_{e}\right)$ is maximal cyclic in $G_{\delta_{0}(e)}$.

(N3) If $e_{1} \cdots e_{n}$ is a loop with image $\left(f_{\bar{e}_{i}}\right)=\operatorname{image}\left(f_{e_{i+1}}\right)$ for $1 \leq i<n$ and $\operatorname{image}\left(f_{\bar{e}_{n}}\right)=\operatorname{image}\left(f_{e_{1}}\right)$, then $\left(f_{\bar{e}_{n}} f_{e_{n}}^{-1}\right) \ldots\left(f_{\bar{e}_{1}} f_{e_{1}}^{-1}\right)$ is the identity automorphism on image $\left(f_{e_{1}}\right)$.

(N4) For each vertex $\iota$ and each maximal cyclic subgroup $C \leq G_{a}$ there is at most one edge $e$ with image $\left(f_{e}\right)=C$ except that there may be two such edges if the pair $\left(G_{u}, C\right)$ is isomorphic to $\left(\mathbf{Z}_{n}, \mathbf{Z}_{n}\right),\left(\mathbf{D}_{2 n+1}, \mathbf{Z}_{2}\right)$, or $\left(\mathbf{A}_{4}, \mathbf{Z}_{3}\right)$.

If $(\Gamma, \mathbf{G})$ is a graph of groups satisfying the normalized conditions then $(\Gamma, \mathbf{G})$ is said to be in canonical form provided that it has the form

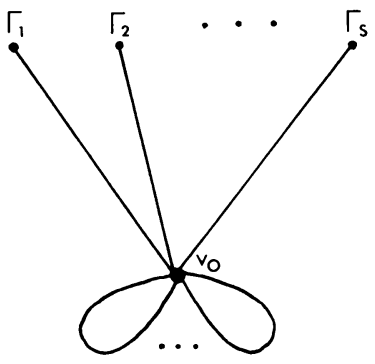

where each $\Gamma_{k}$ is a subgraph of $\Gamma$ which contains no edges with trivial edge group and no trivial edges (an edge is trivial if it is not a loop and its edge-to-vertex 
monomorphism is surjective), the vertex group $G_{a_{0}}$ is trivial, and each edge not contained in one of the $\Gamma_{k}$ 's has trivial edge group. This form is really canonical in the sense that, except for the choice of basepoint in each $\Gamma_{k}$, it uniquely determines the homeomorphism type of its corresponding orbifold handlebody.

PROPOSITION 1.1. If either $V$ is the orbifold quotient of $V_{g}$ under an orientation-preserving finite group action, or $V$ is homeomorphic to $V\left(\Gamma_{0}, \mathbf{G}_{0}\right)$ where $\left(\Gamma_{0}, \mathbf{G}_{0}\right)$ satisfies the normalized conditions, then $V$ is homeomorphic to $V(\Gamma, \mathbf{G})$ for a graph of groups $(\Gamma, \mathbf{G})$ in canonical form.

Proof. Suppose that $\phi$ is a $G$-action on $V_{g}$. By the Equivariant Dehn Lemma $[\mathbf{M Y}, \mathbf{E 2}]$ there is an essential properly imbedded disk $D$ in $V_{g}$ such that for each $x \in G$ either $x D=D$ or $x D \cap D=\varnothing$. Repeating this we find an equivariant disjoint collection $\Delta$ of such disks so that each component of the complement $V_{g}^{\prime}$ of the interior of a regular neighborhood of $\Delta$ is a 3-ball. The action of the stabilizer of each 3-ball is equivalent to a linear action on that 3-ball by the generalization of the Smith conjecture [T]. (In many cases-such as when $G$ is cyclic or has odd order-the only subgroups of $G$ which can act on $B^{3}$ are cyclic and the Smith conjecture itself [MB] applies.) Therefore the quotient of each 3-ball in $V_{g}^{\prime}$ is an orbifold 0-handle and it follows that $V_{g} / \phi$ has a decomposition into orbifold 0handles and orbifold 1-handles. This decomposition has a dual graph of groups $\left(\Gamma_{0}, G_{0}\right)$ (taking a vertex for each 0-handle, an edge for each 1-handle, and using the corresponding stabilizers as the vertex and edge groups) which is readily seen to satisfy the normalized conditions. Thus $V_{g} / \phi$ is homeomorphic to $V\left(\Gamma_{0}, \mathbf{G}_{0}\right)$. It remains to show that $V\left(\Gamma_{0}, \mathbf{G}_{0}\right)$ is homeomorphic to $V(\Gamma, \mathbf{G})$, where $(\Gamma, \mathbf{G})$ is a graph of groups in canonical form.

To systematically transform $\left(\Gamma_{0}, \mathbf{G}_{0}\right)$ so that it is in canonical form, we will use three basic modifications on a graph of groups $\left(\Gamma^{\prime}, \mathbf{G}^{\prime}\right)$. The first of these is called a collapse of $e$, where $e$ is a trivial edge in $\left(\Gamma^{\prime}, \mathbf{G}^{\prime}\right)$ (that is $e$ is not a loop and $G_{e}$ is isomorphic to $G_{\delta_{0} e}, \delta_{0} e$ being the initial vertex of $e$ ). Such a collapse is described by:

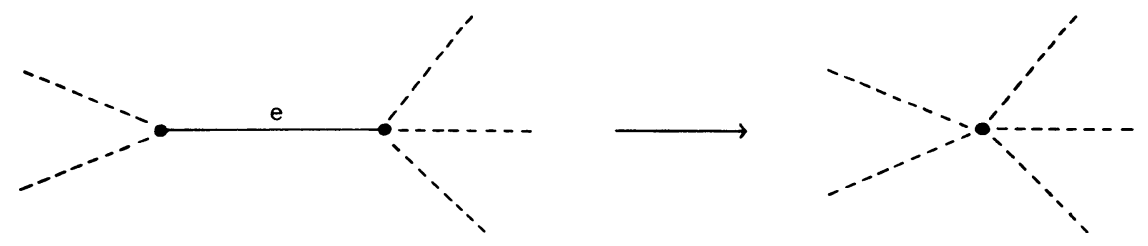

The next modification is called a switch and has the effect of moving an edge $e$ with $G_{e}=1$ across another edge $e^{\prime}$.

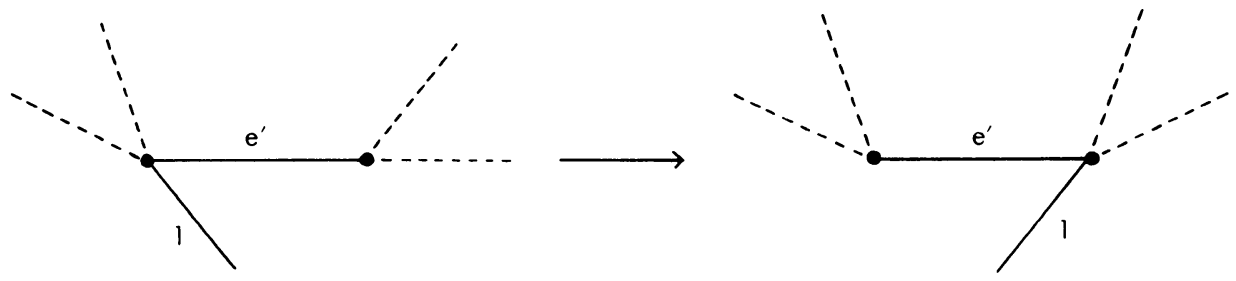


The final modification is called a trivial expansion and its effect is
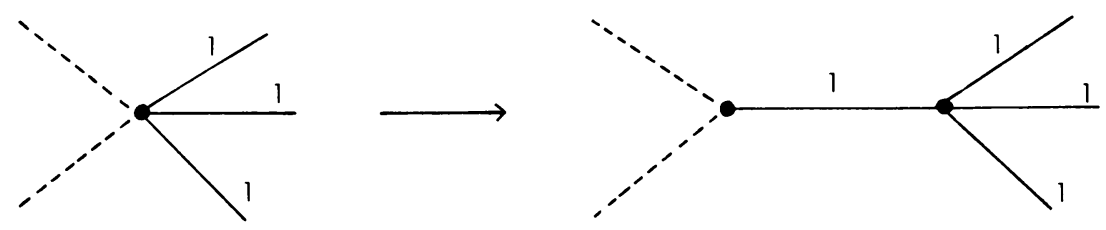

Each of these modifications is readily seen to preserve the normalized conditions and to preserve the homeomorphism type of $V\left(\Gamma^{\prime}, \mathbf{G}^{\prime}\right)$. For the latter one may observe that a collapse coalesces an orbifold 1-handle into an adjacent orbifold 0handle, a trivial expansion adds a 1-handle into an orbifold 0-handle, and a switch is a composition of the addition of one orbifold 1-handle with the coalescing of another.

We are now ready to describe how to transform $\left(\Gamma_{0}, \mathbf{G}_{0}\right)$ into a graph of groups in canonical form. The first step is to perform a succession of collapses so as to eliminate all trivial edges in $\left(\Gamma_{0}, \mathbf{G}_{0}\right)$. The components of the complement of \{ele is an edge with $\left.G_{e}=1\right\}$ in the resulting graph of groups are denoted by $\Gamma_{1}, \ldots, \Gamma_{s}$. For each $k=1, \ldots, s$ choose a basepoint $u_{k} \in \Gamma_{k}$. Now perform switches so that the resulting graph of groups has the property that each edge $e$ with $G_{e}=1$ has $\delta_{0} e=u_{k}$ for some $k$. Next, for each $k=1, \ldots, s$ we do a trivial expansion so that there is exactly one edge $e$ with $G_{e}=1$ and $\delta_{0} e=u_{k}$. Finally, we successively collapse trivial edges $e$ (which have $G_{e}=G_{\delta_{0 e}}=1$ ) until there is exactly one vertex $u_{0}$ with $G_{\omega_{0}}=1$. The graph of groups $(\Gamma, \mathbf{G})$ thus obtained is in canonical form and $V\left(\Gamma_{0}, \mathbf{G}_{0}\right)$ is homeomorphic with $V(\Gamma, \mathbf{G})$.

In the proof of Theorem 1.3 we will refer to the following lemma from orbifold covering space theory - its proof is directly analogous to the corresponding covering space lemma.

LEMMA 1.2. Let $V^{1}$ and $V^{2}$ be homeomorphic manifolds. For $k=1$, 2 let $\phi_{k}$ be a $G$-action on $V^{k}$ and let $\nu_{k}:\left(V^{k}, z_{k}\right) \rightarrow\left(V^{k} / \phi_{k}, w_{k}\right)$ be its associated orbifold covering map. If

$$
H:\left(V^{1} / \phi_{1}, w_{1}\right) \rightarrow\left(V^{2} / \phi_{2}, w_{2}\right)
$$

and

$$
h:\left(V^{1}, z_{1}\right) \rightarrow\left(V^{2}, z_{2}\right)
$$

are homeomorphisms such that $\nu_{2} h=H \nu_{1}$ then

$$
\rho_{\nu_{2}} H_{\#}=\mu(h) \rho_{\nu_{1}},
$$

where $\rho_{\nu_{k}}: \Pi_{1}^{\text {orb }}\left(V^{k} / \phi_{k}, w_{k}\right) \rightarrow \operatorname{Homeo}\left(V^{k}\right)$ is the covering translation homomorphism associated with $\nu_{k}$ and where $\mu(h)$ is defined by $\mu(h) \alpha=h \alpha h^{-1}$ whenever $\alpha \in \operatorname{Homeo}\left(V^{1}\right)$.

Let $g$ be a positive integer and let $G$ be a finite group. We consider the set of pairs $((\Gamma, \mathbf{G}), \lambda)$, where $(\Gamma, \mathbf{G})$ is a graph of groups satisfying the normalized 
conditions with $\chi(\Gamma, \mathbf{G})=(1-g) /|G|$, and where $\lambda: \Pi_{1}^{\text {orb }}(V(\Gamma, \mathbf{G})) \rightarrow G$ is a finite injective epimorphism (that is, $\operatorname{ker}(\lambda)$ is torsion free). (When such an epimorphism exists we say that $(\Gamma, \mathbf{G})$ is $G$-admissible.) We define an equivalence relation on this set of pairs by setting

$$
((\Gamma, \mathbf{G}), \lambda) \equiv\left(\left(\Gamma^{\prime}, \mathbf{G}^{\prime}\right), \lambda^{\prime}\right)
$$

if and only if there is an orbifold homeomorphism $H: V\left(\Gamma^{\prime}, \mathbf{G}^{\prime}\right) \rightarrow V(\Gamma, \mathbf{G})$ such that $\lambda^{\prime}=\lambda H_{\#}$. Now define $\Delta\left(G, V_{g}\right)$ to be the set of equivalence classes $[(\Gamma, \mathbf{G}), \lambda]$ under this equivalence relation.

THEOREM 1.3. The set $\mathscr{E}\left(G, V_{g}\right)$ of equivalence classes of orientation preserving effective $G$-actions on the handlebody $V_{g}$ is in 1-1 correspondence with the set $\Delta\left(G, V_{g}\right)$.

ProOF. We shall first define a function $\Theta: \Delta\left(G, V_{g}\right) \rightarrow \mathscr{E}\left(G, V_{g}\right)$ and then show that it is a bijection.

Let $[(\Gamma, \mathbf{G}), \lambda]$ be an element of $\Delta\left(G, V_{g}\right)$. Choose an orbifold covering $\nu: V \rightarrow$ $V(\Gamma, \mathbf{G})$ with $\operatorname{im}\left(\nu_{\#}\right)=\operatorname{ker}(\lambda)$. Since $V$ is an orbifold handlebody with torsion free fundamental group, it must be a handlebody of genus

$$
1-|G| \chi(V(\Gamma, \mathbf{G}))=1-|G| \chi(\Gamma, \mathbf{G})=g .
$$

Choose a homeomorphism $f: V \rightarrow V_{g}$ and, for $x \in G$, define

$$
\phi(x)=\mu(f) \rho_{\nu}\left(x_{0}\right), \quad \text { where } \lambda\left(x_{0}\right)=x .
$$

(Recall that $\rho_{\nu}$ is the covering translation homomorphism for $\nu$.) Then $\phi: G \rightarrow$ Homeo $^{+}\left(V_{g}\right)$ is a $G$-action. Set

$$
\Theta([(\Gamma, \mathbf{G}), \lambda])=[\phi],
$$

where $[\phi]$ denotes the equivalence class of the action $\phi$.

We now verify that $\Theta$ is a well-defined function. Suppose that $\left[\left(\Gamma^{\prime}, \mathbf{G}^{\prime}\right), \lambda^{\prime}\right]=$ $[(\Gamma, \mathbf{G}), \lambda]$ in $\Delta\left(G, V_{g}\right)$. By definition there is an orbifold homeomorphism $H: V\left(\Gamma^{\prime}, \mathbf{G}^{\prime}\right) \rightarrow V(\Gamma, \mathbf{G})$ such that $\lambda H_{\#}=\lambda^{\prime}$. Let $\nu^{\prime}: V^{\prime} \rightarrow V\left(\Gamma^{\prime}, \mathbf{G}^{\prime}\right)$ be an orbifold covering with $\operatorname{im}\left(\nu_{\#}^{\prime}\right)=\operatorname{ker}\left(\lambda^{\prime}\right)$ and, using this, choose $f^{\prime}$ and $\phi^{\prime}$ in the same fashion as $f$ and $\phi$ were chosen previously. Since $H_{\#}\left(\operatorname{ker}\left(\lambda^{\prime}\right)\right)=\operatorname{ker}(\lambda), H$ lifts to a basepoint preserving homeomorphism $h: V^{\prime} \rightarrow V$. Applying Lemma 1.2 we have

$$
\begin{aligned}
\phi \lambda & =\mu(f) \rho_{\nu}=\mu(f) \mu(h) \rho_{\nu^{\prime}} H_{\#}^{-1} \\
& =\mu(f) \mu(h) \mu\left(f^{\prime-1}\right) \phi^{\prime} \lambda^{\prime} H_{\#}^{-1}=\mu\left(f h f^{\prime-1}\right) \phi^{\prime} \lambda
\end{aligned}
$$

and hence, since $\lambda$ is surjective,

$$
\phi=\mu\left(f h f^{\prime-1}\right) \phi^{\prime} .
$$

Therefore $\phi$ is equivalent to $\phi^{\prime}$, which shows that $\Theta$ is well defined.

To show that $\Theta$ is surjective, consider a $G$-action $\phi$ on $V_{g}$. By Proposition 1.1 there is a graph of groups $(\Gamma, \mathbf{G})$ in canonical form and an orbifold homeomorphism $H: V_{g} / \phi \rightarrow V(\Gamma, \mathbf{G})$. Let $\nu: V_{g} \rightarrow V_{g} / \phi$ be the quotient map. Then $\nu$ and $H \nu$ are orbifold covering maps. Define $\lambda$ to be $\phi^{-1} \rho_{H \nu}$ and observe that $[(\Gamma, \mathbf{G}), \lambda]$ is an element of $\Delta\left(G, V_{g}\right)$. To compute $\Theta([(\Gamma, \mathbf{G}), \lambda])$ we use the covering $H \nu$ and take 
$f=1_{V_{g}}$; these choices imply that $\Theta([(\Gamma, \mathbf{G}), \lambda])=\left[\phi^{\prime}\right]$ where $\phi^{\prime} \lambda=\rho_{H \nu}$. Since $\lambda$ is surjective it follows that $\phi^{\prime}=\phi$ and that $\Theta$ is surjective.

In order to verify that $\Theta$ is injective, suppose that $\Theta([(\Gamma, \mathbf{G}), \lambda])$ equals $\Theta\left(\left[\left(\Gamma^{\prime}, \mathbf{G}^{\prime}\right), \lambda^{\prime}\right]\right)$. Choose covering maps $\nu: V \rightarrow V(\Gamma, \mathbf{G})$ and $\nu^{\prime}: V^{\prime} \rightarrow V\left(\Gamma^{\prime}, \mathbf{G}^{\prime}\right)$ and homeomorphisms $f: V \rightarrow V_{g}$ and $f^{\prime}: V^{\prime} \rightarrow V_{g}$ in order to obtain the $G$ actions $\phi$ and $\phi^{\prime}$ with $\Theta([(\Gamma, \mathbf{G}), \lambda])=[\phi]$ and $\Theta\left(\left[\left(\Gamma^{\prime}, \mathbf{G}^{\prime}\right), \lambda^{\prime}\right]\right)=\left[\phi^{\prime}\right]$. Recall that $\phi \lambda=\mu(f) \rho_{\nu}$ and $\phi^{\prime} \lambda^{\prime}=\mu\left(f^{\prime}\right) \rho_{\nu^{\prime}}$. Since $[\phi]=\left[\phi^{\prime}\right]$ there is $k \in \operatorname{Homeo}^{+}\left(V_{g}\right)$ so that $\mu(k) \phi^{\prime}=\phi$. Let $F, F^{\prime}$, and $K$ be homeomorphisms which make the following diagram commute:

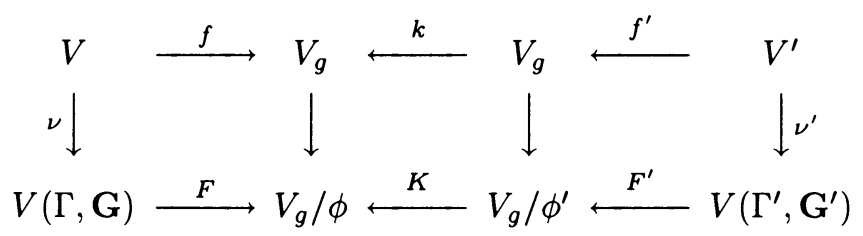

Using Lemma 1.2 we have

$$
\begin{aligned}
\lambda\left(F^{-1} K F^{\prime}\right)_{\#} & =\phi^{-1} \mu(f) \rho_{\nu}\left(F^{-1} K F^{\prime}\right)_{\#}=\phi^{-1} \mu(f) \mu\left(f^{-1} k f^{\prime}\right) \rho_{\nu^{\prime}} \\
& =\phi^{-1} \mu(k) \mu\left(f^{\prime}\right) \rho_{\nu^{\prime}}=\phi^{-1} \mu(k) \phi^{\prime} \lambda^{\prime}=\lambda^{\prime} .
\end{aligned}
$$

This shows that $[(\Gamma, \mathbf{G}), \lambda]$ equals $\left[\left(\Gamma^{\prime}, \mathbf{G}^{\prime}\right), \lambda^{\prime}\right]$, completing the proof of the theorem.

COROLlARY 1.4. For a fixed $g>1$ there are finitely many finite group actions (effective and orientation-preserving) on $V_{g}$ up to equivalence.

Proof. Suppose that the finite group $G$ acts on $V_{g}$ where $g>1$. Then $|G| \leq$ $12(g-1)$ [Z, MMZ, §7]. This implies that, up to isomorphism, there are only finitely many groups which act on $V_{g}$. It now suffices to show that one finite group $G$ arises as an action on $V_{g}$ in only finitely many ways up to equivalence. Thus, by Theorem 1.3, we need only show that $\Delta\left(G, V_{g}\right)$ is finite for a fixed group $G$.

Suppose that $[(\Gamma, \mathbf{G}), \lambda] \in \Delta\left(G, V_{g}\right)$, where $(\Gamma, \mathbf{G})$ is a graph of groups in canonical form whose preferred basepoint is $v_{0}$ (we may assume this by Proposition 1.1). In referring to the graph of groups $(\Gamma, \mathbf{G})$ we use notation of $[\mathbf{M M Z}, \S 1]$; in particular, the graph $\Gamma$ has twice as many "edges" as its geometric realization $|\Gamma|$. Let $T$ be a maximal tree in $\Gamma$ and choose an orientation $\mathscr{O}$ of $T$ so that $V(\Gamma)-\left\{\iota_{0}\right\}=\left\{\delta_{0} e \mid e \in \mathscr{O}\right\}$. (This orientation satisfies the property that there is a unique oriented path in $T$ from each vertex of $\Gamma$ to $\sim_{0}$.) We have

$$
\begin{aligned}
& \chi(T, \mathbf{G})=\frac{1}{\left|G_{\prime_{0}}\right|}+\sum_{\rho \in \mathscr{G}^{\prime}}\left(\frac{1}{\left|G_{\delta_{0} \rho}\right|}-\frac{1}{\left|G_{e}\right|}\right) \\
& \leq 1+\sum_{e \in \mathscr{G}^{\prime}} \frac{1}{\left|G_{\rho}\right|}\left(-\frac{1}{2}\right) \quad(\text { since }(\Gamma, \mathbf{G}) \text { is in canonical form } \\
& \text { it follows that } \left.\left|G_{e}\right| /\left|G_{\delta_{0}}\right| \leq \frac{1}{2}\right) \\
& \leq 1-\frac{1}{2} \sum_{\rho \in \mathscr{G}^{\prime}} \frac{1}{|G|} \\
& \leq 1-|E(\Gamma)| \frac{1}{|G|} \text {. }
\end{aligned}
$$


Therefore,

$$
\begin{aligned}
\chi(\Gamma, \mathbf{G}) & =\chi(T, \mathbf{G})-\sum_{|e| \in E(|\Gamma|)-E(|T|)} \frac{1}{\left|G_{e}\right|} \\
& \leq 1-\frac{|E(\Gamma)|}{|G|}-\frac{1}{2} \sum_{e \in E(\Gamma)-E(T)} \frac{1}{|G|} \\
& \leq 1-\frac{|E(\Gamma)|}{|G|}-\frac{1}{2} \frac{|E(\Gamma)-E(T)|}{|G|} .
\end{aligned}
$$

It follows that

$$
\begin{aligned}
|E(\Gamma)| & \leq 2|E(T)|+|E(\Gamma)-E(T)| \leq 2|G|(1-\chi(\Gamma, \mathbf{G})) \\
& \leq 2(|G|+g-1), \quad \text { since } \chi(\Gamma, \mathbf{G})=(1-g) /|G| .
\end{aligned}
$$

Since the number of edges $|E(\Gamma)|$ is bounded, it follows that there are only finitely many graphs $\Gamma$ for which there exist $\mathbf{G}$ and $\lambda$ such that $(\Gamma, \mathbf{G})$ has canonical form and $[(\Gamma, \mathbf{G}), \lambda] \in \Delta\left(G, V_{g}\right)$. As the orders of the vertex and edge groups in $\mathbf{G}$ are bounded by $|G|$ there can only be finitely many such $(\Gamma, \mathbf{G})$ 's. For a fixed $(\Gamma, \mathbf{G})$, since $\Pi_{1}(\Gamma, \mathbf{G})$ is finitely generated, there are only finitely many epimorphisms $\lambda: \Pi_{1}(\Gamma, \mathbf{G}) \rightarrow G$ and therefore $\Delta\left(G, V_{g}\right)$ is finite.

2. Equivalence of prime order cyclic group actions. Let $\mathbf{v}=(r, s, t)$ be an ordered triple of nonnegative integers and let $p$ be a prime number. Consider the graph of groups $(\Gamma(\mathbf{v}), \mathbf{G}(\mathbf{v}))=$

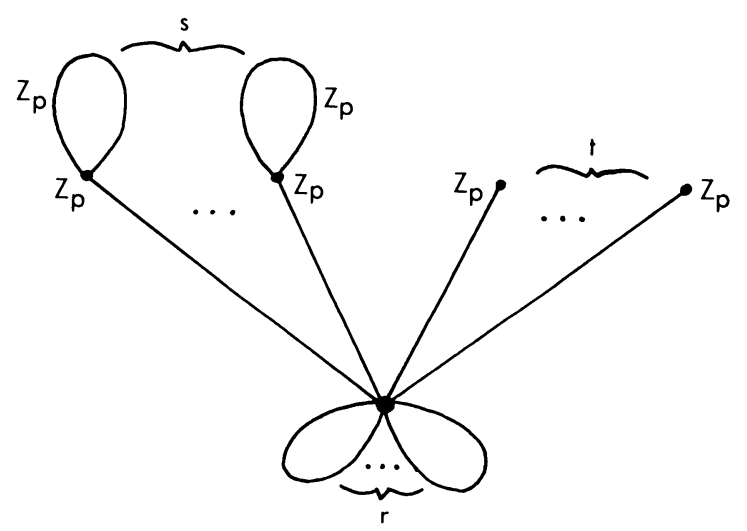

where each unlabelled edge has trivial edge group. Notice that $(\Gamma(\mathbf{v}), \mathbf{G}(\mathbf{v}))$ is in canonical form, and it determines a handlebody orbifold $V(\Gamma(\mathbf{v}), \mathbf{G}(\mathbf{v}))$. As we will see below, the quotient of any $\mathbf{Z}_{p}$-action on a handlebody is an orbifold of this type. Note that the fundamental group $\Pi_{1}^{\text {orb }}(V(\Gamma(\mathbf{v}), \mathbf{G}(\mathbf{v})))$ is a free product

$$
\left(*_{r} \mathbf{Z}\right) *\left(*_{s}\left(\mathbf{Z}_{p} \times \mathbf{Z}\right)\right) *\left(*_{t} \mathbf{Z}_{p}\right)
$$

and therefore it has generators

$$
\left\{a_{i} \mid 1 \leq i \leq r\right\} \cup\left\{b_{j}, c_{j} \mid 1 \leq j \leq s\right\} \cup\left\{d_{k} \mid 1 \leq k \leq t\right\}
$$

subject to the relations $b_{j}^{p}=d_{k}^{p}=1$ and $\left[b_{j}, c_{j}\right]=1$. 
Proposition 2.1. If $\alpha \in \operatorname{Aut}\left(\Pi_{1}^{\text {orb }}(V(\Gamma(\mathbf{v}), \mathbf{G}(\mathbf{v})))\right)$ then $\alpha=H_{\#}$ for an orientation-preserving homeomorphism $H: V(\Gamma(\mathbf{v}), \mathbf{G}(\mathbf{v})) \rightarrow V(\Gamma(\mathbf{v}), \mathbf{G}(\mathbf{v}))$ if and only if

$$
\begin{aligned}
\alpha\left(b_{j}\right) & =x_{j} b_{\sigma(j)}^{\varepsilon_{j}} x_{j}^{-1}, \\
\alpha\left(c_{j}\right) & =x_{j} b_{\sigma(j)}^{n_{j}} c_{\sigma(j)}^{\varepsilon_{j}} x_{j}^{-1}, \quad \text { and } \\
\alpha\left(d_{k}\right) & =y_{k} d_{\tau(k)}^{\delta_{k}} y_{k}^{-1}
\end{aligned}
$$

for some $x_{j}$ and $y_{k}$ in $\Pi_{1}^{\text {orb }}(V(\Gamma(\mathbf{v}), \mathbf{G}(\mathbf{v}))), \sigma \in \sum_{s}, \tau \in \sum_{t}$, and $\varepsilon_{j}$ and $\delta_{k}$ in $\{+1,-1\}\left(\sum_{l}\right.$ denotes the permutation group on l letters $)$.

ProOF. Let $R$ be the subgroup of $\operatorname{Aut}\left(\Pi_{1}^{\text {orb }}(V(\Gamma(\mathbf{v}), \mathbf{G}(\mathbf{v})))\right)$ consisting of those automorphisms which are induced by homeomorphisms of $V(\Gamma(\mathbf{v}), \mathbf{G}(\mathbf{v}))$.

In $[\mathbf{F}-\mathbf{R}]$ it is shown that a generating set for $\operatorname{Aut}\left(\Pi_{1}^{\text {orb }}(V(\Gamma(\mathbf{v}), \mathbf{G}(\mathbf{v})))\right)$ is

$$
S=\left\{\rho_{j i}(x), \lambda_{j i}(x), \mu_{j i}(x), \omega_{i j}, \sigma_{i}, \phi_{i}\right\},
$$

where we use the notation of $[\mathbf{M M}, \S 5.1]$ as reproduced below. To set up the indices, we view $V(\Gamma(\mathbf{v}), \mathbf{G}(\mathbf{v}))$ as obtained from a 3-ball by: attaching $r$ 1-handles $V_{1}, \ldots, V_{r}$; boundary connect-summing $s$ copies $V_{r+1}, \ldots, V_{r+s}$ of $V(\Gamma, \mathbf{G})$ where $(\Gamma, \mathbf{G})$ is a graph of groups of the form

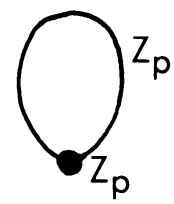

and boundary connect-summing $t$ copies $V_{r+s+1}, \ldots, V_{r+s+t}$ of $V\left(\Gamma^{\prime}, \mathbf{G}^{\prime}\right)$ where $\left(\Gamma^{\prime}, \mathbf{G}^{\prime}\right)$ is a graph of groups with one vertex $a$ and $G_{\iota}=\mathbf{Z}_{p}$. We write $\Pi_{1}^{\text {orb }}\left(V_{i}\right)=$ $G_{i}$ so that $\Pi_{1}^{\text {orb }}(V(\Gamma(\mathbf{v}), \mathbf{G}(\mathbf{v})))=G_{1} * G_{2} * \cdots * G_{r+s+t}$.

Each of the generators in $S$ has its support in one of the free factors $G_{i}$ of $G_{1} * \cdots * G_{r+s+t}$. Their definitions are as follows, where $G_{i}$ is generated by $a_{i}$ for $i \leq r$, by $b_{i}$ and $c_{i}$ for $r+1 \leq i \leq r+s$, and by $d_{i}$ for $r+s+1 \leq i \leq r+s+t$. In each case all nondisplayed generators are left fixed, and the indices $i$ and $j$ are never equal. In the first three cases $x$ is a fixed element of $G_{j}$, and in the last case $\phi$ is a fixed automorphism of $G_{i}$ where $i \geq r+1$.

(1) $\rho_{j i}(x)\left(a_{i}\right)=a_{i} x$

(2) $\lambda_{j i}(x)\left(a_{i}\right)=x^{-1} a_{i}$

(3) $\mu_{j i}(x)(g)=x^{-1} g x$ for all $g \in G_{i}$,

(4) $\omega_{i j}\left(a_{i}\right)=a_{j}, \omega_{i j}\left(a_{j}\right)=a_{i}$ if $i, j \leq r$,

(5) $\omega_{i j}\left(b_{i}\right)=b_{j}, \omega_{i j}\left(c_{i}\right)=c_{j}, \omega_{i j}\left(b_{j}\right)=b_{i}, \omega_{i j}\left(c_{j}\right)=c_{i}$ if $r<i, j \leq r+s$,

(6) $\omega_{i j}\left(d_{i}\right)=d_{j}, \omega_{i j}\left(d_{j}\right)=d_{i}$ if $r+s<i, j$,

(7) $\sigma_{i}\left(a_{i}\right)=a_{i}^{-1}$

(8) $\phi_{i}(g)=\phi(g)$ for all $g \in G_{i}, i \geq r+1$.

From these definitions, each of the generators in the set $S_{1}=S-\left\{\phi_{i}\right\}$ is readily seen to satisfy the condition given in the statement of the proposition. Furthermore, if we make an analogy of $V(\Gamma(\mathbf{v}), \mathbf{G}(\mathbf{v}))$ with a product-with-handles $V$ as defined 
in [MM, §1.2], the proof used in Proposition 5.2.2 of [MM] adapts to show that $S_{1} \subset R$. (The generators $\rho_{j i}(x), \lambda_{j i}(x), \mu_{j i}(x)$ come from "orbifold slides", $\omega_{i j}$ comes from the "interchange" of $V_{i}$ with $V_{j}$, and $\sigma_{i}$ is a "spin" of $V_{i}$ where $i \leq r$.)

Using some relations in $\operatorname{Aut}\left(G_{1} * \cdots * G_{r+s+t}\right)$ (see [MM, $\left.\S 5.1\right]$ ), it is easily seen that $\alpha$ may be written as $\alpha=\alpha_{1} \alpha_{2}$ where $\alpha_{1}$ is in the subgroup generated by $S_{1}$ and $\alpha_{2}=\prod_{i=r+1}^{r+s+t} \phi_{i}$. By the remarks in the previous paragraph, $\alpha_{1}$ satisfies the conditions of the proposition and $\alpha_{1} \in R$. Thus $\alpha \in R$ if and only if $\alpha_{2} \in R$, and $\alpha$ satisfies the conditions given in the proposition if and only if $\alpha_{2}$ satisfies those conditions. In this way we have reduced the proof of the proposition to the case where $\alpha=\alpha_{2}=\prod_{i=r+1}^{r+s+t} \phi_{i}$.

Suppose that $\alpha \in R$. Let $h$ be a homeomorphism of $V(\Gamma(\mathbf{v}), \mathbf{G}(\mathbf{v}))$ with $h_{\#}=$ $\alpha=\prod_{i=r+1}^{r+s+t} \phi_{i}$. Since $\alpha$ leaves each $G_{i}$ invariant, it follows that $h$ leaves each component of the singular set of $V(\Gamma(\mathbf{v}), \mathbf{G}(\mathbf{v}))$ invariant. For each $i$ let $U_{i}$ be a regular neighborhood of the $i$ th component of the singular set. Notice that $U_{i}$ is homeomorphic to $V_{i}$, and that $\left(\left.h\right|_{U_{i}}\right)_{\#}=\left.\alpha\right|_{G_{i}}=\phi_{i}$. If $r+1 \leq i \leq r+s$ then $G_{i}$ is generated by $b_{i}$ and $c_{i}$ and $\phi_{i}\left(b_{i}\right)=b_{i}^{\varepsilon}$ and $\phi_{i}\left(c_{i}\right)=b_{i}^{n} c_{i}^{\varepsilon}$ for some $\varepsilon= \pm 1$ and $n<p$. To see this, consider the inclusion $\partial U_{i} \rightarrow U_{i}$ and the corresponding homomorphism

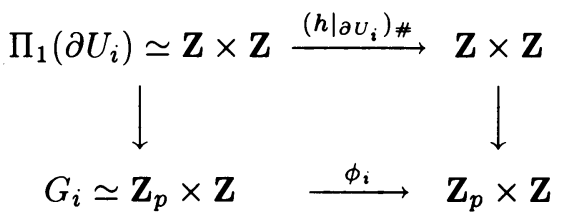

of "group systems" induced by $h$. Since $h$ is orientation-preserving, $\left(\left.h\right|_{\partial U_{i}}\right)_{\#}$ corresponds to a $2 \times 2$ matrix with determinant 1 , and commutativity of the above diagram implies that this matrix has the form $\left[\begin{array}{ll}\varepsilon & n \\ 0 & \varepsilon\end{array}\right]$ which implies that $\phi_{i}$ has the claimed form. In a similar way, if $r+s+1 \leq i \leq r+s+t$ then consideration of the inclusion mapping $\operatorname{Fr}_{V_{i}}\left(U_{i}\right) \rightarrow U_{i}$ leads to the conclusion that $\phi_{i}\left(d_{i}\right)=d_{i}^{ \pm 1}$. In either case, we see that $\phi_{i}$ satisfies the conditions of the proposition and this implies that $\alpha=\prod_{i=r+1}^{r+s+t} \phi_{i}$ satisfies those conditions.

Now suppose that $\alpha=\prod_{i=r+1}^{r+s+t} \phi_{i}$ satisfies the conditions given in the proposition. Then each $\phi_{i}$ satisfies those conditions and it is readily seen that each such automorphism is induced by a homeomorphism of $V_{i}$ and of $V(\Gamma(\mathbf{v}), \mathbf{G}(\mathbf{v})$ ). (If $r+1 \leq i \leq r+s$, then $\phi_{i}$ is induced by a power of a Dehn twist about a meridional orbifold 2-disk in $V_{i}$ possibly composed with an involution which induces a reflection on the singular circle of $V_{i}$. If $r+s+1 \leq i \leq r+s+t$, then $\phi_{i}$ is induced by the identity map or by an involution which induces a reflection on the singular arc of $V_{i}$.) Since each $\phi_{i}$ is in $R$, it follows that $\alpha \in R$ and the proof of the proposition is complete.

LEMMA 2.2. If $\mathbf{v} \neq(1,0,0)$ and if $\lambda_{1}$ and $\lambda_{2}$ are finite injective epimorphisms from $\Pi_{1}(\Gamma(\mathbf{v}), \mathbf{G}(\mathbf{v}))$ to $\mathbf{Z}_{p}$, then there is an automorphism $\alpha$ such that $\lambda_{2} \alpha=\lambda_{1}$.

ProOF. Using the description of $\Pi_{1}^{\text {orb }}(V(\Gamma(\mathbf{v}), \mathbf{G}(\mathbf{v})))$ given above, we define $\alpha$ by considering two cases.

Case 1: $s+t>0$. In this case define $\alpha$ on generators by

$$
\alpha\left(a_{i}\right)=a_{i} w_{i}, \quad \alpha\left(b_{j}\right)=b_{j}^{n_{j}}, \quad \alpha\left(c_{j}\right)=b_{j}^{l_{j}} c_{j}^{ \pm 1}, \quad \alpha\left(d_{k}\right)=d_{k}^{m_{k}},
$$


where $w_{i}, n_{j}, l_{j}$, and $m_{k}$ are chosen in the following ways:

$$
\begin{gathered}
w_{i} \in\left\langle b_{1}\right\rangle \quad \text { or } \quad w_{i} \in\left\langle d_{1}\right\rangle, \quad \text { and } \quad \lambda_{2}\left(w_{i}\right)=\lambda_{1}\left(a_{i}\right)-\lambda_{2}\left(a_{i}\right) ; \\
n_{j}=\lambda_{1}\left(b_{j}\right) / \lambda_{2}\left(b_{j}\right) ; \quad l_{j}=\left(\lambda_{1}\left(c_{j}\right)-\lambda_{2}\left(c_{j}\right)\right) / \lambda_{2}\left(b_{j}\right) ; \quad m_{k}=\lambda_{1}\left(d_{k}\right) / \lambda_{2}\left(d_{k}\right) .
\end{gathered}
$$

Since $\alpha$ respects the relations on $\Pi_{1}(\Gamma(\mathbf{v}), \mathbf{G}(\mathbf{v}))$, it defines a homomorphism. It is evident that $\alpha$ is surjective. Now $\Pi_{1}(\Gamma(\mathbf{v}), \mathbf{G}(\mathbf{v}))$ is a Hopfian group since it is the free product of Hopfian groups, so $\alpha$ must be an isomorphism. A straightforward check shows that $\lambda_{2} \alpha=\lambda_{1}$ on the generators and therefore $\lambda_{2} \alpha=\lambda_{1}$ as claimed.

Case 2: $s=t=0$. In this case $\Pi_{1}(\Gamma(\mathbf{v}), \mathbf{G}(\mathbf{v}))$ is freely generated by $\left\{a_{1}, \ldots, a_{r}\right\}$ so we may identify $\Pi_{1}(\Gamma(\mathbf{v}), \mathbf{G}(\mathbf{v}))$ with the free group $F_{r}$ on the generators $\left\{a_{1}, \ldots\right.$, $\left.a_{r}\right\}$. Let $\lambda: F_{r} \rightarrow \mathbf{Z}_{p}$ be the epimorphism defined by $\lambda\left(a_{i}\right)=1$ for all $i$. We shall show that there are automorphisms $\alpha_{1}$ and $\alpha_{2}$ such that $\lambda_{1} \alpha_{1}=\lambda$ and $\lambda_{2} \alpha_{2}=\lambda$. Then $\alpha=\alpha_{2} \alpha_{1}^{-1}$ will give the desired automorphism since $\lambda_{2} \alpha=\lambda_{2} \alpha_{2} \alpha_{1}^{-1}=$ $\lambda \alpha_{1}^{-1}=\lambda_{1}$.

To define $\alpha_{1}$, choose integers $k$ and $j$ between 1 and $r$ so that $\lambda_{1}\left(a_{k}\right) \neq 0$ and $j$ is not equal to $k$. Define $\alpha_{1}$ by

$$
\alpha_{1}=\left(\prod_{i \neq k} \rho_{k i}\left(a_{k}^{l_{i}}\right)\right) \rho_{j k}\left(a_{j}^{l}\right),
$$

where $l_{i}=\left(1-\lambda_{1}\left(a_{i}\right)\right) / \lambda_{1}\left(a_{k}\right)$ and $l=1-\lambda_{1}\left(a_{k}\right)$. Then for $i \neq k$,

$$
\lambda_{1} \alpha_{1}\left(a_{i}\right)=\lambda_{1}\left(a_{i} a_{k}^{l_{i}}\right)=\lambda_{1}\left(a_{i}\right)+l_{i} \lambda_{1}\left(a_{k}\right)=1=\lambda\left(a_{i}\right)
$$

and

$$
\begin{aligned}
\lambda_{1} \alpha_{1}\left(a_{k}\right) & =\lambda_{1}\left(a_{k}\left(a_{j} a_{k}^{l_{j}}\right)^{l}\right)=\lambda_{1}\left(a_{k}\right)+l\left(\lambda_{1}\left(a_{j}\right)+l_{j} \lambda_{1}\left(a_{k}\right)\right) \\
& =\lambda_{1}\left(a_{k}\right)+l=1=\lambda\left(a_{k}\right) .
\end{aligned}
$$

Thus $\lambda_{1} \alpha_{1}=\lambda$.

To obtain $\alpha_{2}$ we just repeat the definition of the previous paragraph, using $\alpha_{2}$ in place of $\alpha_{1}$.

REMARK. A straightforward application of Proposition 2.1 shows that the automorphism $\alpha$ constructed in the proof of Lemma 2.2 may be realized by an orbifold homeomorphism of $V(\Gamma(\mathbf{v}), \mathbf{G}(\mathbf{v}))$ provided that $\lambda_{i}\left(b_{j}\right)= \pm \lambda_{2}\left(b_{j}\right)$ (so that $n_{j}= \pm 1$ ) and that $\lambda_{1}\left(d_{k}\right)= \pm \lambda_{2}\left(d_{k}\right)$ (so that $m_{k}= \pm 1$ ).

For a fixed prime $p$ and positive integer $g$ we shall consider the set of all 3-tuples $\mathbf{v}=(r, s, t)$ of nonnegative integers which satisfy

$$
p(r+s)+(p-1)(t-1)=g .
$$

Let $\mathbf{Z}_{p}^{*}$ denote the multiplicative group of the field $\mathbf{Z}_{p}$ and let $\sum_{k}$ denote the permutation group on $k$ letters. We define an equivalence relation on $\left(\mathbf{Z}_{p}^{*}\right)^{s}$ by setting

$$
\left(x_{1}, \ldots, x_{s}\right) \sim\left(x_{1}^{\prime}, \ldots, x_{s}^{\prime}\right) \quad \text { if and only if } \quad x_{j}^{\prime}=\varepsilon_{j} x_{\sigma(j)}
$$

for some $\varepsilon_{j} \in\{ \pm 1\}$ and $\sigma \in \Sigma_{s}$. Let $[\mathbf{x}]$ denote the equivalence class of $\mathbf{x}=$ $\left(x_{1}, \ldots, x_{s}\right) \in\left(\mathbf{Z}_{p}^{*}\right)^{s}$ under this relation. Similarly, on $\left(\mathbf{Z}_{p}^{*}\right)^{t}$, we set

$$
\mathbf{y}=\mathbf{y}^{\prime} \quad \text { if and only if } y_{k}^{\prime}=\delta_{k} y_{\tau(k)}
$$


for some $\delta_{k} \in\{ \pm 1\}$ and $\tau \in \Sigma_{t}$ and we denote the equivalence class of $\mathbf{y}$ by [y]. We consider the data set

$$
\mathscr{D}\left(\mathbf{Z}_{p}, V_{g}\right)=\{(\mathbf{v},[\mathbf{x}],[\mathbf{y}])\}
$$

where $\mathbf{v}=(r, s, t),[\mathbf{x}]$ and $[\mathbf{y}]$ satisfy the conditions given above.

THEOREM 2.3. For every prime $p$ and integer $g>1$ there is a 1-1 correspondence of $\mathscr{E}\left(\mathbf{Z}_{p}, V_{g}\right)$ with $\mathscr{D}\left(\mathbf{Z}_{p}, V_{g}\right)$.

ProOF. We shall describe a bijection $\Lambda: \mathscr{D}\left(\mathbf{Z}_{p}, V_{g}\right) \rightarrow \Delta\left(\mathbf{Z}_{p}, V_{g}\right)$. By Theorem $1.3, \Theta \Lambda$ will be a $1-1$ correspondence between $\mathscr{D}\left(\mathbf{Z}_{p}, V_{g}\right)$ and $\mathscr{E}\left(\mathbf{Z}_{p}, V_{g}\right)$.

To define $\Lambda$, let $(\mathbf{v},[\mathbf{x}],[\mathbf{y}]) \in \mathscr{D}\left(\mathbf{Z}_{p}, V_{g}\right)$ be given, where

$$
\mathbf{x}=\left(x_{1}, \ldots, x_{s}\right) \in\left(\mathbf{Z}_{p}^{*}\right)^{s} \quad \text { and } \quad \mathbf{y}=\left(y_{1}, \ldots, y_{t}\right) \in\left(\mathbf{Z}_{p}^{*}\right)^{t}
$$

Consider the graph of groups $(\Gamma(\mathbf{v}), \mathbf{G}(\mathbf{v}))$ (which is in canonical form), and its associated handlebody orbifold $V(\Gamma(\mathbf{v}), \mathbf{G}(\mathbf{v}))$. Observe that

$$
\begin{aligned}
\chi(\Gamma(\mathbf{v}), \mathbf{G}(\mathbf{v})) & =1-r-s-t(1-1 / p) \\
& =(1-(p(r+s)+(t-1)(p-1))) / p \\
& =(1-g) / p .
\end{aligned}
$$

As described above, $\Pi_{1}^{\text {orb }}(V(\Gamma(\mathbf{v}), \mathbf{G}(\mathbf{v}))) \simeq\left(*_{r} \mathbf{Z}\right) *\left(*_{s}\left(\mathbf{Z}_{p} \times \mathbf{Z}\right)\right) *\left(*_{t} \mathbf{Z}_{p}\right)$ and it is generated by $\left\{a_{i} \mid 1 \leq i \leq r\right\} \cup\left\{b_{j}, c_{j} \mid 1 \leq j \leq s\right\} \cup\left\{d_{k} \mid 1 \leq k \leq t\right\}$, the successive elements generating the corresponding factors. Define $\lambda: \Pi_{1}^{\text {orb }}(V(\Gamma(\mathbf{v}), \mathbf{G}(\mathbf{v}))) \rightarrow$ $\mathbf{Z}_{p}$ by

$$
\begin{aligned}
& \lambda\left(a_{i}\right)=1 \quad \text { for } i=1, \ldots, r, \\
& \left.\begin{array}{l}
\lambda\left(b_{j}\right)=x_{j} \\
\lambda\left(c_{j}\right)=1
\end{array}\right\} \quad \text { for } j=1, \ldots, s, \\
& \lambda\left(d_{k}\right)=y_{k} \quad \text { for } k=1, \ldots, t .
\end{aligned}
$$

It is apparent that $\lambda$ is an epimorphism; furthermore it is finite injective since each element of finite order is conjugate into a subgroup generated by some $b_{j}$ or some $d_{k}$. It follows that $[(\Gamma(\mathbf{v}), \mathbf{G}(\mathbf{v})), \lambda] \in \Delta\left(\mathbf{Z}_{p}, V_{g}\right)$ and we define $\Lambda(\mathbf{v},[\mathbf{x}],[\mathbf{y}])$ to be $[(\Gamma(\mathbf{v}), \mathbf{G}(\mathbf{v})), \lambda]$.

In the previous paragraph, if $\mathbf{x}^{\prime} \in\left(\mathbf{Z}_{p}^{*}\right)^{s}$ and $\mathbf{y}^{\prime} \in\left(\mathbf{Z}_{p}^{*}\right)^{t}$ are used in place of $\mathbf{x}$ and $\mathbf{y}$ then we obtain a finite injective epimorphism $\lambda^{\prime}: \Pi_{1}^{\text {orb }}(V(\Gamma(\mathbf{v}), \mathbf{G}(\mathbf{v}))) \rightarrow \mathbf{Z}_{p}$. If, in addition, $\left[\mathbf{x}^{\prime}\right]=[\mathbf{x}]$ and $\left[\mathbf{y}^{\prime}\right]=[\mathbf{y}]$ then there are $\sigma \in \Sigma_{s}, \tau \in \Sigma_{t}, \varepsilon_{j} \in\{ \pm 1\}$, and $\delta_{k} \in\{ \pm 1\}$ such that $x_{j}^{\prime}=\varepsilon_{j} x_{\sigma(j)}$ and $y_{k}^{\prime}=\delta_{k} y_{\tau(k)}$. Let $\alpha$ be the automorphism of $\Pi_{1}^{\text {orb }}(V(\Gamma(\mathbf{v}), \mathbf{G}(\mathbf{v})))$ which satisfies: $\alpha\left(a_{i}\right)=a_{i}, \alpha\left(b_{j}\right)=\left(b_{\sigma(j)}\right)^{\varepsilon_{j}}, \alpha\left(c_{j}\right)=$ $c_{\sigma(j)}$, and $\alpha\left(d_{k}\right)=\left(d_{\tau(k)}\right)^{\delta_{k}}$. It may now be seen that $\lambda \alpha=\lambda^{\prime}$ and that, by Proposition 2.1, $\alpha=H_{\#}$ for some orbifold homeomorphism $H$ of $V(\Gamma(\mathbf{v}), \mathbf{G}(\mathbf{v}))$. Therefore $[(\Gamma(\mathbf{v}), \mathbf{G}(\mathbf{v})), \lambda]=\left[(\Gamma(\mathbf{v}), \mathbf{G}(\mathbf{v})), \lambda^{\prime}\right]$; this implies that the function $\Lambda$ is well defined.

We now show that $\Lambda$ is injective. Let $(\mathbf{v},[\mathbf{x}],[\mathbf{y}])$ and $\left(\mathbf{v}^{\prime},\left[\mathbf{x}^{\prime}\right],\left[\mathbf{y}^{\prime}\right]\right)$ be elements of $\mathscr{D}\left(\mathbf{Z}_{p}, V_{g}\right)$ for which

$$
\Lambda(\mathbf{v},[\mathbf{x}],[\mathbf{y}])=[(\Gamma(\mathbf{v}), \mathbf{G}(\mathbf{v})), \lambda] \quad \text { and } \quad \Lambda\left(\mathbf{v}^{\prime},\left[\mathbf{x}^{\prime}\right],\left[\mathbf{y}^{\prime}\right]\right)=\left[\left(\Gamma\left(\mathbf{v}^{\prime}\right), \mathbf{G}\left(\mathbf{v}^{\prime}\right)\right), \lambda^{\prime}\right]
$$

are equal. Since $\Pi_{1}(\Gamma(\mathbf{v}), \mathbf{G}(\mathbf{v}))$ is isomorphic to $\Pi_{1}\left(\Gamma\left(\mathbf{v}^{\prime}\right), \mathbf{G}\left(\mathbf{v}^{\prime}\right)\right)$ we must have $\mathbf{v}=\mathbf{v}^{\prime}$. Let $H$ be an orbifold homeomorphism of $V(\Gamma(\mathbf{v}), \mathbf{G}(\mathbf{v}))$ such that 
$\lambda H_{\#}=\lambda^{\prime}$. By Proposition 2.1, $H_{\#}\left(b_{j}\right)$ is conjugate to $b_{\sigma(j)}^{\varepsilon_{j}}$ for some $\sigma$ and $\varepsilon_{j}$. Thus, in $\mathbf{Z}_{p}$ we have

$$
x_{j}^{\prime}=\lambda^{\prime}\left(b_{j}\right)=\lambda H_{\#}\left(b_{j}\right)=\varepsilon_{j} x_{\sigma(j)} .
$$

This shows that $[\mathbf{x}]=\left[\mathbf{x}^{\prime}\right]$. It follows by a similar argument that $[\mathbf{y}]=\left[\mathbf{y}^{\prime}\right]$ and we conclude that $\Lambda$ is injective.

To complete the proof, it remains to show that $\Lambda$ is surjective. Suppose that $[(\Gamma, \mathbf{G}), \lambda]$ is an element of $\Delta\left(\mathbf{Z}_{p}, V_{g}\right)$. Using Proposition 1.1 we may modify $(\Gamma, \mathbf{G})$ to a graph of groups $\left(\Gamma^{\prime}, \mathbf{G}^{\prime}\right)$ which is in canonical form. Since $\left(\Gamma^{\prime}, \mathbf{G}^{\prime}\right)$ is $\mathbf{Z}_{p^{-}}$ admissible, each vertex and edge group of $\mathbf{G}^{\prime}$ is either trivial or $\mathbf{Z}_{p}$. Since $\left(\Gamma^{\prime}, \mathbf{G}^{\prime}\right)$ has no trivial edges, it follows that each component $\Gamma_{k}$ of $\left(\Gamma^{\prime}, \mathbf{G}^{\prime}\right)$ is either a $\mathbf{Z}_{p}$ vertex or a $\mathbf{Z}_{p}$ loop; thus $\left(\Gamma^{\prime}, \mathbf{G}^{\prime}\right)=(\Gamma(\mathbf{v}), \mathbf{G}(\mathbf{v}))$ for some $\mathbf{v}=(r, s, t)$. Using Euler characteristics it is evident that

$$
g=p(r+s)+(p-1)(t-1)
$$

By Proposition 1.1 there is an orbifold homeomorphism

$$
F: V(\Gamma, \mathbf{G}) \rightarrow V(\Gamma(\mathbf{v}), \mathbf{G}(\mathbf{v})) .
$$

Now define a finite injective epimorphism $\lambda^{\prime}: \Pi_{1}^{\text {orb }}(V(\Gamma(\mathbf{v}), \mathbf{G}(\mathbf{v}))) \rightarrow \mathbf{Z}_{p}$ by

$$
\lambda^{\prime}\left(a_{i}\right)=1, \quad \lambda^{\prime}\left(b_{j}\right)=\lambda F_{\#}^{-1}\left(b_{j}\right), \quad \lambda^{\prime}\left(c_{j}\right)=1, \quad \lambda^{\prime}\left(d_{k}\right)=\lambda F_{\#}^{-1}\left(d_{k}\right) .
$$

By Lemma 2.2, there is an automorphism $\alpha$ such that $\lambda^{\prime}=\lambda F_{\#}^{-1} \alpha$. This automorphism $\alpha$ must be realizable on $V(\Gamma(\mathbf{v}), \mathbf{G}(\mathbf{v}))$ since the remark following Lemma 2.2 is applicable. It follows that

$$
\begin{aligned}
& \Lambda\left(\mathbf{v},\left[\left(\lambda F_{\#}^{-1}\left(b_{j}\right)\right)_{j}\right],\left[\left(\lambda F_{\#}^{-1}\left(d_{k}\right)\right)_{k}\right]\right) \\
& \quad=\left[(\Gamma(\mathbf{v}), \mathbf{G}(\mathbf{v})), \lambda^{\prime}\right]=\left[(\Gamma(\mathbf{v}), \mathbf{G}(\mathbf{v})), \lambda F_{\#}^{-1}\right]=[(\Gamma, \mathbf{G}), \lambda]
\end{aligned}
$$

which completes the proof.

COROLlaRY 2.4. Let $p$ be a prime and let $g>1$.

(a) If $p=2$ then the number of equivalence classes of $\mathbf{Z}_{p}$-actions on $V_{g}$ is $(g+2)(g+4) / 8$ when $g$ is even, and $(g+3)(g+5) / 8$ when $g$ is odd.

(b) If $p>2$ then the number of equivalence classes of $\mathbf{Z}_{p}$-actions on $V_{g}$ is

$$
\sum_{k=-[(1-g) / p]}^{[g /(p-1)]}\left(\begin{array}{c}
p k-g+(p-1) / 2 \\
(p-3) / 2
\end{array}\right)\left(\begin{array}{c}
g-k(p-1)+(p-1) / 2 \\
(p-1) / 2
\end{array}\right) .
$$

PROOF. Suppose that $\mathbf{v}=(r, s, t)$ is a 3-tuple of integers satisfying

$$
r \geq 0, \quad s \geq 0, \quad t \geq 0, \quad \text { and } \quad g=p(r+s)+(p-1)(t-1) .
$$

If $R=r+s$ then $R \equiv g(\bmod p-1)$ and so there is a positive integer $k$ with $R=g-k(p-1)$. Observe that

$$
t=1+(g-p R) /(p-1)=p k-g+1 .
$$

Since $R \geq 0$ and $t \geq 0$ it follows that

$$
(g-1) / p \leq k \leq g /(p-1) .
$$


Moreover, if $k$ is a nonnegative integer satisfying (B) and $s$ is a nonnegative integer less than or equal to $R=g-k(p-1)$ (note that $R \geq 0$ by the second inequality of (B)) then setting $r=R-s$ and $t=p k-g+1$ gives a 3-tuple $(r, s, t)$ which satisfies (A). This shows that the set of all $\mathbf{v}=(r, s, t)$ satisfying (A) is in 1-1 correspondence with the pairs of integers $(k, s)$, where $k$ satisfies (B) and $0 \leq s \leq g-k(p-1)$.

Let $N_{s}$ be the number of equivalence classes in $\left(\mathbf{Z}_{p}^{*}\right)^{s} / \sim$ where $\sim$ is the equivalence relation given in defining $\mathscr{D}\left(\mathbf{Z}_{p}, V_{g}\right)$. Similarly define $N_{t}$. By Theorem 2.3 and the results of the previous paragraph we see that

$$
\begin{aligned}
\left|\mathscr{E}\left(\mathbf{Z}_{p}, V_{g}\right)\right| & \left.=\left|\mathscr{D}\left(\mathbf{Z}_{p}, V_{g}\right)\right|\right)=\sum_{\substack{(r, s, t) \\
\text { satisfying (A) }}} N_{s} N_{t} \\
& =\sum_{k=-[(1-g) / p]} \sum_{s=0}^{g-k(p-1)} N_{s} N_{p k-g+1} .
\end{aligned}
$$

Now consider the case where $p=2$. Observe that $N_{s}=N_{t}=1$ in this case. Therefore

$$
\left|\mathscr{E}\left(\mathbf{Z}_{2}, V_{g}\right)\right|=\sum_{k=-[(1-g) / 2]}^{g} g-k+1
$$

and by separately considering the cases where $g$ is even or odd, a straightforward computation leads to (a).

Now suppose $p>2$. Observe that $N_{s}=\left|\left(\mathbf{Z}_{(p-1) / 2}\right)^{s} / \Sigma_{s}\right|$, where $\Sigma_{s}$ acts on $\left(\mathbf{Z}_{(p-1) / 2}\right)^{s}$ by the standard permutation of factors. In turn, there is a 1-1 correspondence between $\left(\mathbf{Z}_{(p-1) / 2}\right)^{s} / \Sigma_{s}$ and the set $S(s,(p-1) / 2)$ of $(p-1) / 2$-tuples of nonnegative integers $\left(a_{1}, \ldots, a_{(p-1) / 2}\right)$ with $\sum a_{i}=s$. (Here $\left(a_{1}, \ldots, a_{(p-1) / 2}\right)$ corresponds to an $s$-tuple $\left(x_{1}, \ldots, x_{s}\right)$ for which exactly $a_{i}$ of the $x_{k}$ 's equal $i$.)

SUBLEMMA. The cardinality of the set $S(s, l)$ of $l$-tuples of nonnegative integers $\left(a_{1}, \ldots, a_{l}\right)$ with $\sum_{i=1}^{l} a_{i}=s$ is $\left(\begin{array}{c}s+l-1 \\ l-1\end{array}\right)$.

PROOF. We induct on $l$; when $l=1$ the result holds. If it holds for $l-1$ then

$$
|S(s, l)|=\sum_{k=0}^{s}|S(k, l-1)|=\sum_{k=0}^{s}\left(\begin{array}{c}
k+l-2 \\
l-2
\end{array}\right)=\left(\begin{array}{c}
s+l-1 \\
l-1
\end{array}\right)
$$

and the proof of the sublemma is complete.

Using the sublemma we now have that

$$
N_{s}=\left(\begin{array}{c}
s+(p-3) / 2 \\
(p-3) / 2
\end{array}\right)
$$

Thus

$$
\begin{aligned}
\left|\mathscr{E}\left(\mathbf{Z}_{p}, V_{g}\right)\right| & =\sum_{k} \sum_{s}\left(\begin{array}{c}
s+(p-3) / 2 \\
(p-3) / 2
\end{array}\right)\left(\begin{array}{c}
t+(p-3) / 2 \\
(p-3) / 2
\end{array}\right) \\
& =\sum_{k}\left(\begin{array}{c}
p k-g+(p-1) / 2 \\
(p-3) / 2
\end{array}\right) \sum_{s=0}^{g-k(p-1)}\left(\begin{array}{c}
s+(p-3) / 2 \\
(p-3) / 2
\end{array}\right) \\
& =\sum_{k}\left(\begin{array}{c}
p k-g+(p-1) / 2 \\
(p-3) / 2
\end{array}\right)\left(\begin{array}{c}
g-k(p-1)+(p-3) / 2+1 \\
(p-3) / 2+1
\end{array}\right) .
\end{aligned}
$$


The proof of the following lemma is directly analogous to the standard argument used in covering space theory.

LEMMA 2.5. Let $\phi: G \rightarrow$ Homeo $^{+}\left(V_{g}\right)$ be an effective $G$-action and let $\nu:\left(V_{g}, z\right)$ $\rightarrow\left(V_{g} / \phi, w\right)$ be the corresponding orbifold covering map. Then the associated exact sequence

$$
1 \rightarrow \Pi_{1}\left(V_{g}, z\right) \stackrel{\nu_{\#}}{\rightarrow} \Pi_{1}^{\text {orb }}\left(V_{g} / \phi, w\right) \stackrel{\phi^{-1} \rho_{\nu}}{\rightarrow} G \rightarrow 1
$$

has abstract kernel $\Psi \phi$. That is $\Psi \phi(x)=\left[\nu_{\#}^{-1} \mu\left(x^{\prime}\right) \nu_{\#}\right] \in \operatorname{Out}\left(\Pi_{1}\left(V_{g}, z\right)\right)$, where $x^{\prime}$ is any element of $\Pi_{1}^{\mathrm{orb}}\left(V_{g} / \phi, w\right)$ with $\phi^{-1} \rho_{\nu}\left(x^{\prime}\right)=x \in G$.

PROPOSITION 2.6. Let $g>1$. If $\phi_{1}$ and $\phi_{2}$ are $\mathbf{Z}_{p}$-actions on $V_{g}$ with the same quotient type, then $\phi_{2}$ is equivalent to a $\mathbf{Z}_{p}$-action $\phi_{2}^{\prime}$ which is homotopic to $\phi_{1}$.

PROOF. Let $\nu_{k}: V_{g} \rightarrow V_{g} / \phi_{k}$ be the orbifold covering maps corresponding to $\phi_{1}$ and $\phi_{2}$. As in Lemma 2.5 these determine short exact sequences

$$
\varepsilon_{k}: 1 \rightarrow F_{g} \stackrel{i_{k}}{\rightarrow} \Pi_{1}^{\text {orb }}\left(V_{g} / \phi_{k}\right) \stackrel{\lambda_{k}}{\rightarrow} \mathbf{Z}_{p} \rightarrow 1
$$

where $i_{k}=\nu_{k} \#$ and $\lambda_{k}=\phi_{k}^{-1} \rho_{k}$ (recall that $\rho_{k}$ is the covering translation homomorphism corresponding to $\nu_{k}$ ). By Lemma 2.5, $\Psi \phi_{k}$ is the abstract kernel for $\varepsilon_{k}$. We may now apply Lemma 2.2 to find an isomorphism $\alpha$ which makes the following diagram commute:

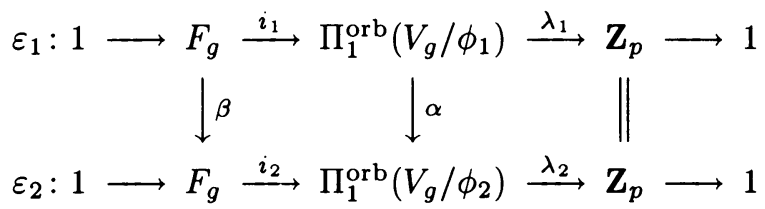

Let $h: V_{g} \rightarrow V_{g}$ be a homeomorphism such that $h_{\#}=\beta$ (Proposition 2.1). Define $\phi_{2}^{\prime}=\mu\left(h^{-1}\right) \phi_{2}$. For $x \in \mathbf{Z}_{p}$ there is $x^{\prime} \in \Pi_{1}^{\text {orb }}\left(V_{g} / \phi_{2}\right)$ with $\lambda_{2}\left(x^{\prime}\right)=x$ and we have:

$$
\Psi \phi_{2}^{\prime}(x)=\Psi\left(h^{-1} \phi_{2}(x) h\right)=h_{\#}^{-1} \Psi \phi_{2}(x) h_{\#}=\beta^{-1}\left(i_{2}^{-1} \mu\left(x^{\prime}\right) i_{2}\right) \beta .
$$

Using the above diagram we obtain the following commutative diagram:

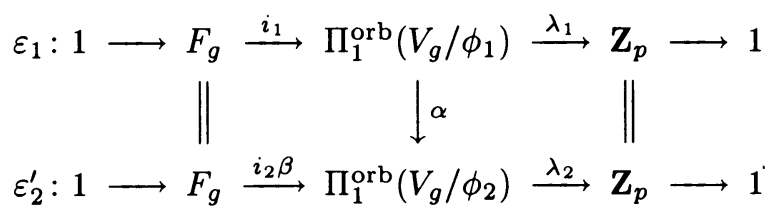

Therefore we have

$$
\beta^{-1}\left(i_{2}^{-1} \mu\left(x^{\prime}\right) i_{2}\right) \beta=\left(i_{2} \beta\right)^{-1} \mu\left(x^{\prime}\right)\left(i_{2} \beta\right)=i_{1}^{-1} \mu\left(\alpha^{-1}\left(x^{\prime}\right)\right) i_{1}=\Psi \phi_{1}(x) .
$$

This shows that $\Psi \phi_{2}^{\prime}=\Psi \phi_{1}$, and consequently $\phi_{2}^{\prime}$ is homotopic to $\phi_{1}$. Since $\phi_{2}^{\prime}$ is equivalent to $\phi_{2}$ by definition, the proof is complete.

3. Strong equivalence of actions of arbitrary groups. In this section we give a characterization of the strong equivalence classes of effective $G$-actions on $V_{g}$ in a given homotopy class. We shall study these strong equivalence classes of actions by relating them to certain group extensions as in Lemma 2.5. The basic connection is given in the following lemma. 
LEMMA 3.1. Let $\phi$ and $\phi^{\prime}$ be effective $G$-actions on $V_{g}$. Then $\phi$ is strongly equivalent to $\phi^{\prime}$ if and only if there is an orbifold homeomorphism $H: V_{g} / \phi \rightarrow V_{g} / \phi^{\prime}$ so that the following diagram commutes:

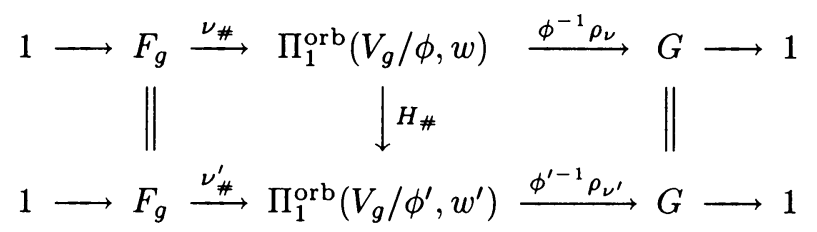

ProOF. First suppose that there exists an orbifold homeomorphism $H$ making the above diagram commute. Since $H_{\#} \nu_{\#}=\nu_{\#}^{\prime}$, it follows that $H$ lifts to a basepoint preserving homeomorphism $h: V_{g} \rightarrow V_{g}$. Therefore

$$
\nu_{\#}^{\prime} h_{\#}=H_{\#} \nu_{\#}=\nu_{\#}^{\prime}
$$

and, since $\nu_{\#}^{\prime}$ is a monomorphism, $h_{\#}$ is the identity automorphism. The asphericity of $V_{g}$ implies that $h$ is homotopic to the identity.

Suppose that $x \in G$ and choose $x^{\prime} \in \Pi_{1}^{\text {orb }}\left(V_{g} / \phi\right)$ such that $\phi(x)=\rho_{\nu}\left(x^{\prime}\right)$. By Lemma $1.2, \mu(h) \phi(x)=\mu(h) \rho_{\nu}\left(x^{\prime}\right)=\rho_{\nu^{\prime}} H_{\#}\left(x^{\prime}\right)$. Using the commutativity of the diagram, $\rho_{\nu^{\prime}} H_{\#}\left(x^{\prime}\right)=\phi^{\prime} \phi^{-1} \rho_{\nu}\left(x^{\prime}\right)=\phi^{\prime} \phi^{-1} \phi(x)=\phi^{\prime}(x)$. Hence $\mu(h) \phi=\phi^{\prime}$ showing that $\phi$ and $\phi^{\prime}$ are strongly equivalent.

For the converse, suppose that $\phi$ is strongly equivalent to $\phi^{\prime}$; that is $\phi^{\prime}=\mu(h) \phi$ for some $h \in$ Homeo $^{+}\left(V_{g}\right)$ which is homotopic to the identity. Let $H: V_{g} / \phi \rightarrow V_{g} / \phi^{\prime}$ be the homeomorphism induced by $h$, and let $\tau$ be the trace of the basepoint $z \in V_{g}$ under a homotopy from the identity to $h$. We may assume that $\tau$ is in the interior of $V_{g}$ and that $\nu^{\prime}(\tau)$ does not meet the exceptional set of $V_{g} / \phi^{\prime}$. Let id $\simeq K$ be an isotopy of $V_{g} / \phi^{\prime}$, whose support misses the exceptional set, such that the trace under this isotopy is $\overline{\nu^{\prime}(\tau)}$. Now lift this isotopy to an isotopy id $\simeq k$ on $V_{g}$. It follows that $k h$ is homotopic to the identity relative to $z$, and that $\mu(k h) \phi=\mu(k) \phi^{\prime}=\phi^{\prime}$. Observe that $(K H)_{\#} \nu_{\#}=\nu_{\#}^{\prime}(k h)_{\#}=\nu_{\#}^{\prime}$ and that $\phi^{\prime-1} \rho_{\nu^{\prime}}(K H)_{\#}=\phi^{\prime-1} \mu(k h) \rho_{\nu}=\phi^{-1} \rho_{\nu}$ (again using Lemma 1.2). This completes the proof.

We now fix a finite group $G$ and a handlebody $V_{g}$. Define an equivalence relation on the set of effective $G$-actions on $V_{g}$ by $\phi \equiv \phi^{\prime}$ if and only if $\phi$ and $\phi^{\prime}$ are homotopic and have the same quotient type. For many groups $G$ this equivalence relation is the same as the homotopy relation between actions (see Lemma 4.1 and the subsequent example). In what follows we consider a fixed equivalence class $C$ under the relation $\equiv$. The equivalence class $C$ determines an abstract kernel $\eta: G \rightarrow \operatorname{Out}\left(F_{g}\right)$. Furthermore, by Proposition 1.1 there is a graph of groups $(\Gamma, \mathbf{G})$ in canonical form such that $V(\Gamma, \mathbf{G})$ is orbifold homeomorphic to $V_{g} / \phi$ for every $G$-action $\phi \in C$.

In the remainder of this section we will use the symbol $E(\Gamma, \mathbf{G})$ to denote the fundamental group $\Pi_{1}^{\text {orb }}(V(\Gamma, \mathbf{G}))$ where $(\Gamma, \mathbf{G})$ is a graph of groups satisfying the normalized conditions. Consider the set of exact sequences of the form

$$
\varepsilon: 1 \rightarrow F_{g} \stackrel{i}{\rightarrow} E(\Gamma, \mathbf{G}) \stackrel{\lambda}{\rightarrow} G \rightarrow 1
$$


which correspond to the abstract kernel $\eta$ determined by $C$. Define an equivalence relation on this set by setting $\varepsilon \equiv \varepsilon^{\prime}$ if and only if there is an orbifold homeomorphism $F: V(\Gamma, \mathbf{G}) \rightarrow V(\Gamma, \mathbf{G})$ making the following diagram commute:

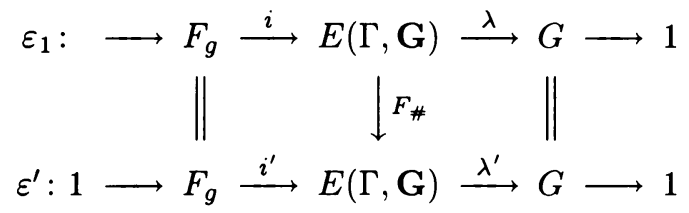

Denote by $\mathscr{S} \mathscr{E} x t((\Gamma, \mathbf{G}), \eta)$ the set of equivalence classes of extensions under this relation.

LEMMA 3.2. There is a 1-1 correspondence between the set $\mathscr{S} \mathscr{E}(C)$ of strong equivalence classes of $G$-actions in $C$ and $\mathscr{S} \mathscr{E} x t((\Gamma, \mathbf{G}), \eta)$.

PROOF. We define a function

$$
\Omega: \mathscr{S} \mathscr{E} x t((\Gamma, \mathbf{G}), \eta) \rightarrow \mathscr{S} \mathscr{E}(C)
$$

as follows. Let

$$
\varepsilon: 1 \rightarrow F_{g} \stackrel{i}{\rightarrow} E(\Gamma, \mathbf{G}) \stackrel{\lambda}{\rightarrow} G \rightarrow 1
$$

be an extension as given above, whose equivalence class is $[\varepsilon] \in \mathscr{S} \mathscr{E} x t((\Gamma, \mathbf{G}), \eta)$. Let $\nu: V_{g} \rightarrow V(\Gamma, \mathbf{G})$ be a covering map with $\nu_{\#}=i$. (To obtain this we choose any covering $\nu^{\prime}: V_{g} \rightarrow V(\Gamma, \mathbf{G})$ with $\operatorname{im}\left(\nu_{\#}^{\prime}\right)=\operatorname{im}(i)$, then find a homeomorphism $k$ so that $k_{\#}=\nu_{\#}^{\prime-1} i$ and put $\nu=\nu^{\prime} k$.) If $\rho_{\nu}$ is the covering translation homomorphism corresponding to $\nu$ then let $\phi=\rho_{\nu} \lambda^{-1}$; this is a $G$-action on $V_{g}$. Let $\nu_{\phi}: V_{g} \rightarrow$ $V_{g} / \phi$ be the quotient covering map. There is a natural orbifold homeomorphism $H: V_{g} / \phi \rightarrow V(\Gamma, \mathbf{G})$ so that $H \nu_{\phi}=\nu$. Using Lemma 1.2 we obtain the following commutative diagram:

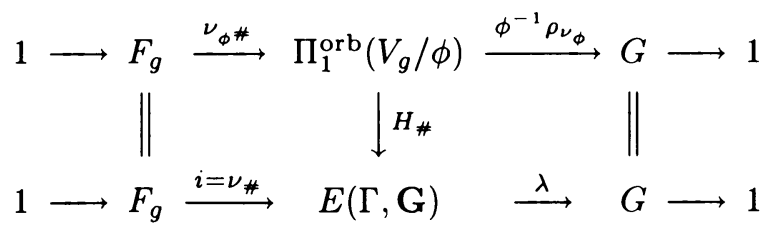

Since the two rows in this diagram have the same abstract kernel, it follows from Lemma 2.5 that $\Psi \phi=\eta$. Thus $\phi \in C$. Define $\Omega([\varepsilon])$ to be the strong equivalence class of the action $\phi$.

We now check to see that $\Omega$ is well defined. Suppose that $[\varepsilon]=\left[\varepsilon^{\prime}\right]$ in

$$
\mathscr{P} \mathscr{E}_{x t}((\Gamma, \mathbf{G}), \eta)
$$


Choosing $\nu$ and $\nu^{\prime}$ as above, we obtain actions $\phi$ and $\phi^{\prime}$ in $C$. We have the following commutative diagram:

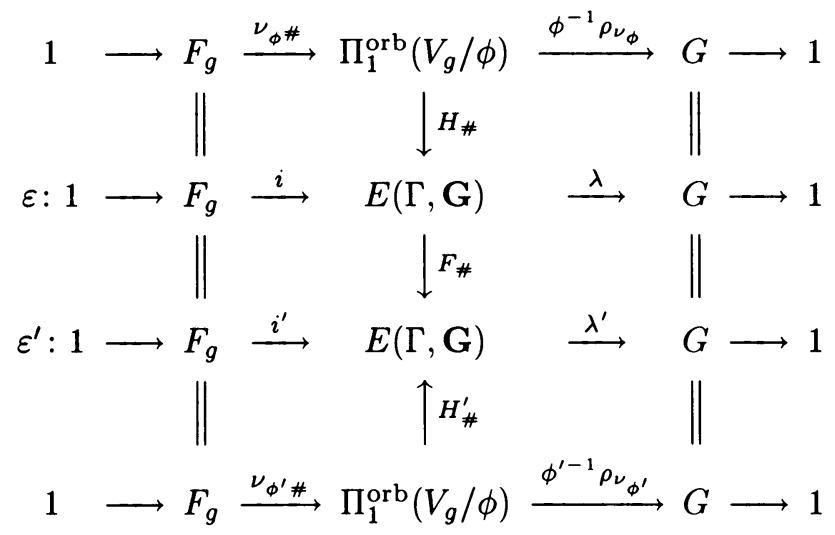

By Lemma 3.1, $\phi$ is strongly equivalent to $\phi^{\prime}$.

Let $\phi \in C$. This implies that there is an orbifold homeomorphism $H: V_{g} / \phi \rightarrow$ $V(\Gamma, \mathbf{G})$ and we obtain an extension $\varepsilon$ :

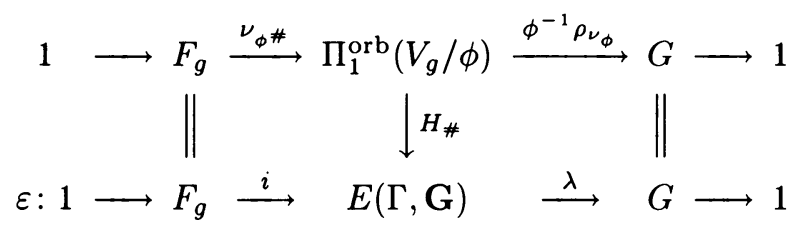

Taking $\nu$ to the $H \nu_{\phi}$ we have

$$
\rho_{\nu} \lambda^{-1}=\rho_{\nu}\left(\phi^{-1} \rho_{\nu_{\phi}} H_{\#}^{-1}\right)^{-1}=\left(\rho_{\nu_{\phi}} H_{\#}^{-1}\right)\left(\phi^{-1} \rho_{\nu_{\phi}} H_{\#}^{-1}\right)^{-1}=\phi,
$$

which shows that $\phi \in \Omega([\varepsilon])$ and that $\Omega$ is surjective.

To show that $\Omega$ is injective, suppose that $\Omega([\varepsilon])=\Omega\left(\left[\varepsilon^{\prime}\right]\right)$. Using Lemma 3.1 we obtain the following commutative diagram:

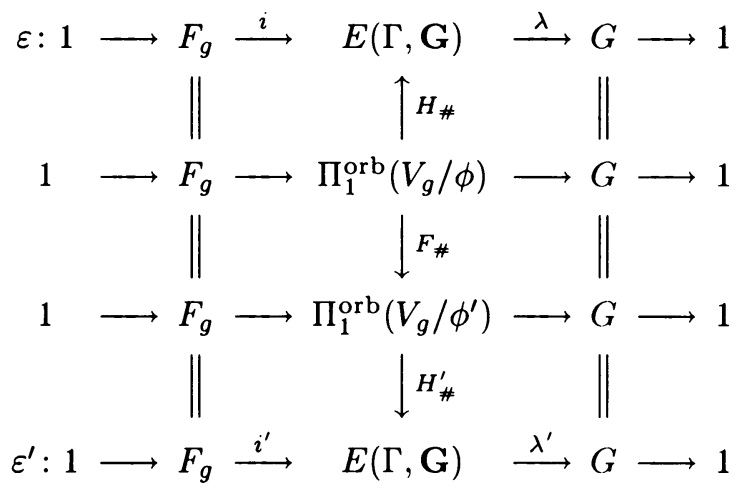

This shows that $[\varepsilon]=\left[\varepsilon^{\prime}\right]$ and completes the proof of the theorem.

Let $R$ be the subgroup of $\operatorname{Aut}(E(\Gamma, \mathbf{G}))$ consisting of automorphisms which may be realized by homeomorphisms of $V(\Gamma, \mathbf{G})$. 
THEOREM 3.3. Let $g$ be greater than 1 . The set $\mathscr{S} \mathscr{E}(C)$ of strong equivalence classes of $G$-actions in $C$ are in 1-1 correspondence with the set of right cosets $R \backslash \operatorname{Aut}(E(\Gamma, \mathbf{G}))$. More precisely, an exact sequence

$$
\varepsilon_{0}: 1 \rightarrow F_{g} \stackrel{i_{0}}{\rightarrow} E(\Gamma, \mathbf{G}) \stackrel{\lambda_{0}}{\rightarrow} G \rightarrow 1
$$

determines a bijection $\Phi_{0}: R \backslash \operatorname{Aut}(E(\Gamma, \mathbf{G})) \rightarrow \mathscr{S} \mathscr{E}(C)$. Furthermore, the equivalence class in $\mathscr{E}\left(G, V_{g}\right)$ corresponding to $\Phi_{0}(R \beta)$ is $\Theta\left(\left[(\Gamma, \mathbf{G}), \lambda_{0} \beta^{-1}\right]\right)$.

Proof. Fix the extension $\varepsilon_{0}: 1 \rightarrow F_{g} \stackrel{i_{0}}{\rightarrow} E(\Gamma, \mathbf{G}) \stackrel{\lambda_{0}}{\rightarrow} G \rightarrow 1$ with abstract kernel $\eta$ (which is determined by $C$ ).

Define a function $\Phi_{0}^{\prime}$ from $R \backslash \operatorname{Aut}(E(\Gamma, \mathbf{G}))$ to $\mathscr{S} \mathscr{E} x t((\Gamma, \mathbf{G}), \eta)$ by sending $R \beta$ (where $\beta \in \operatorname{Aut}(E(\Gamma, \mathbf{G}))$ ) to $\left[\varepsilon_{\beta}\right]$, where the extension $\varepsilon_{\beta}$ is obtained from $\varepsilon_{0}$ as indicated in the following commutative diagram:

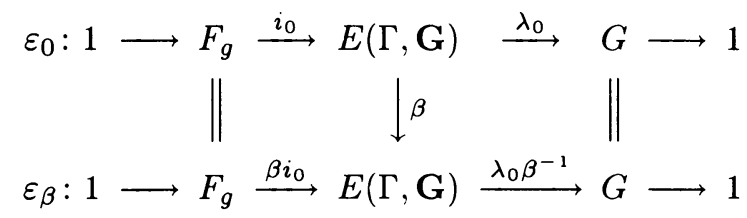

The function $\Phi_{0}$ is now defined as the composition $\Phi_{0}^{\prime} \Omega$, where $\Omega$ is the bijection given in Lemma 3.2.

We first show that $\Phi_{0}^{\prime}$ is well defined. If $R \beta=R \beta^{\prime}$ then $\beta^{\prime}=H_{\#} \beta$ where $H_{\#} \in R$. We obtain the following diagram:

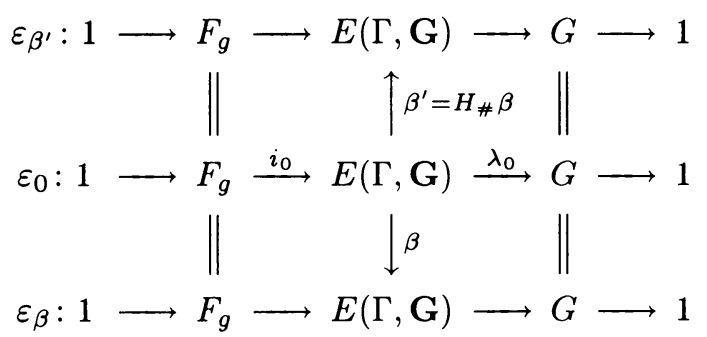

This diagram implies that $\left[\varepsilon_{\beta}\right]=\left[\varepsilon_{\beta^{\prime}}\right]$ and hence the map from $R \backslash \operatorname{Aut}(E(\Gamma, \mathbf{G}))$ to $\mathscr{S} \mathscr{E}_{x<}((\Gamma, \mathbf{G}), \eta)$ is well defined.

Suppose that $\left[\varepsilon_{\beta}\right]=\left[\varepsilon_{\beta^{\prime}}\right]$. Consequently there exists an $H_{\#} \in R$ filling in the following commutative diagram:

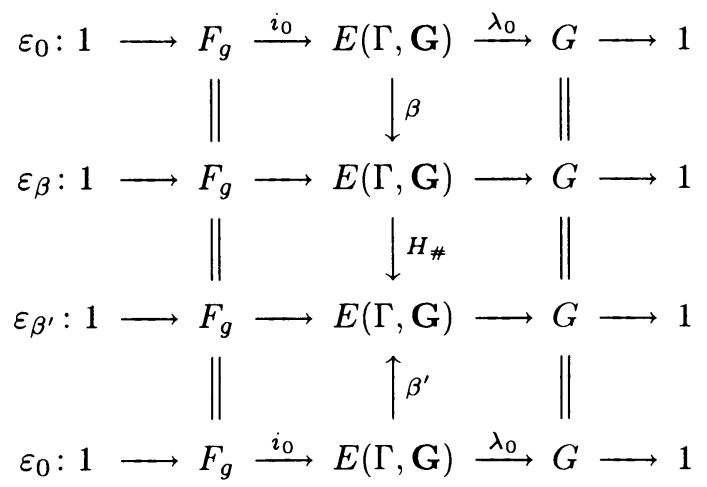


Since $g>1$, the group of cocycles $Z^{1}\left(G\right.$, Cent $\left.\left(F_{g}\right)\right)$ vanishes, implying that $\beta^{\prime-1} H_{\#} \beta$ is the identity automorphism (this is a basic fact in group extension theory; see, for instance, $[\mathrm{G}]$ ). Thus $R \beta^{\prime}=R \beta$ so that the function $\Phi_{0}^{\prime}$ (and also $\left.\Phi_{0}\right)$ must be injective.

Let $[\varepsilon] \in \mathscr{S} \mathscr{E} x t((\Gamma, \mathbf{G}), \eta)$. Since the cohomology group $H^{2}\left(G, \operatorname{Cent}\left(F_{g}\right)\right)$ vanishes, there is $\beta \in \operatorname{Aut}(E(\Gamma, \mathbf{G}))$ making the following diagram commute (this may also be found in $[\mathbf{G}])$ :

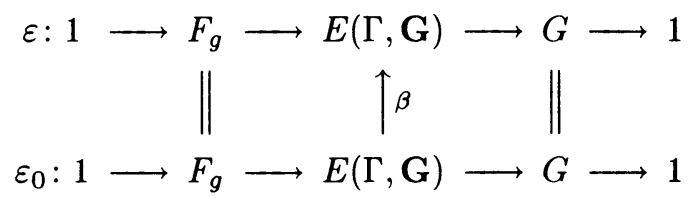

Evidently $R \beta$ gets mapped to $[\varepsilon]$ showing that the function $\Phi_{0}^{\prime}$ is surjective.

To verify the last statement of the theorem, suppose that $R \beta$ is a coset in $R \backslash \operatorname{Aut}(E(\Gamma, \mathbf{G}))$. Observe that $\Phi_{0}(R \beta)=\Omega\left(\left[\varepsilon_{\beta}\right]\right)$; if $\phi$ is a $G$-action representing this strong equivalence class then we have the following diagram:

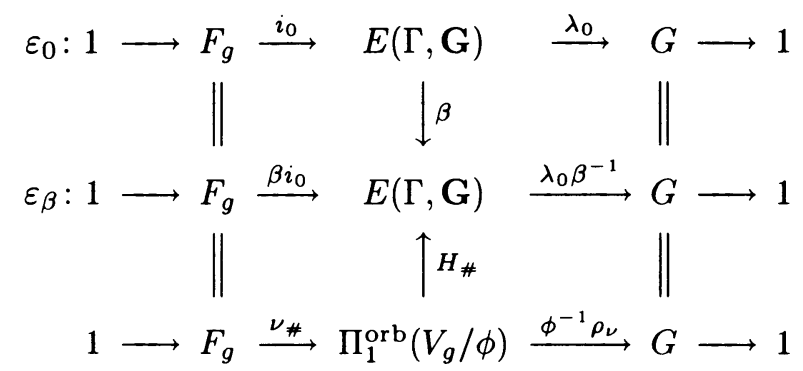

Using this diagram and recalling the definition of $\Theta$, we see that $\Theta\left(\left[(\Gamma, \mathbf{G}), \lambda \beta^{-1}\right]\right)=$ $[\phi]$ in $\mathscr{E}\left(G, V_{g}\right)$.

4. Strong equivalence of prime order cyclic group actions. In this section we apply the main theorem of $\S 3$ to classify and count the strong equivalence classes of $\mathbf{Z}_{p}$-actions on $V_{g}$ in a given homotopy class.

LEMMA 4.1. Let $p$ be a prime number and let $g>1$. If $\phi_{1}$ and $\phi_{2}$ are $\mathbf{Z}_{p^{-}}$ actions on $V_{g}$ which are homotopic, then $\phi_{1}$ and $\phi_{2}$ have the same quotient type.

ProOF. For $k=1$ or $2, V_{g} / \phi_{k}$ is homeomorphic to $V\left(\Gamma_{k}, \mathbf{G}_{k}\right)$, where $\left(\Gamma_{k}, \mathbf{G}_{k}\right)$ is a graph of groups in canonical form (Proposition 1.1). As shown in the proof of Theorem 2.3, a $\mathbf{Z}_{p}$-admissible graph of groups in canonical form must equal $(\Gamma(\mathbf{v}), \mathbf{G}(\mathbf{v}))$ for some $\mathbf{v}=(r, s, t)$ with $g=p(r+s)+(p-1)(t-1)$. Thus each $V_{g} / \phi_{k}$ is homeomorphic to some $V\left(\Gamma\left(\mathbf{v}_{k}\right), \mathbf{G}\left(\mathbf{v}_{k}\right)\right)$. Since $\phi_{1}$ and $\phi_{2}$ are homotopic they determine the same abstract kernel $\mathbf{Z}_{p} \rightarrow \operatorname{Out}\left(F_{g}\right)$ and therefore (since $\operatorname{Cent}\left(F_{g}\right)=$ 1 when $g>1$ ) the extensions

$$
1 \rightarrow F_{g} \rightarrow \Pi_{1}^{\text {orb }}(V(\Gamma(\mathbf{v}), \mathbf{G}(\mathbf{v}))) \rightarrow \mathbf{Z}_{p} \rightarrow 1
$$

are isomorphic. It follows that $\Pi_{1}\left(\Gamma\left(\mathbf{v}_{1}\right), \mathbf{G}\left(\mathbf{v}_{1}\right)\right)$ is isomorphic to $\Pi_{1}\left(\Gamma\left(\mathbf{v}_{2}\right), \mathbf{G}\left(\mathbf{v}_{2}\right)\right)$ and thus (since these are free products whose indecomposable factors are determined by $\left.\left(r_{k}, s_{k}, t_{k}\right)\right) \mathbf{v}_{1}=\mathbf{v}_{2}$ which implies that $\phi_{1}$ and $\phi_{2}$ have the same quotient type. 
The same lines of proof can also be used to show that this lemma holds for $G$ actions, where $G$ is either abelian or has odd order. However it fails in general as shown in the following.

EXAMPLE. Consider the graphs of groups $\left(\Gamma_{1}, \mathbf{G}_{1}\right)$ and $\left(\Gamma_{2}, \mathbf{G}_{2}\right)$ :

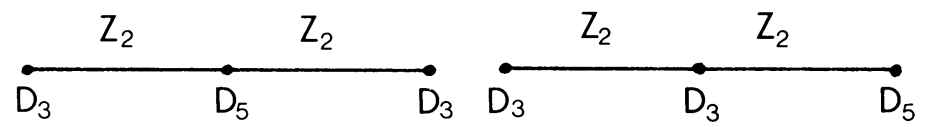

These graphs of groups satisfy the normalized conditions and so they determine handlebody orbifolds $V\left(\Gamma_{1}, \mathbf{G}_{1}\right)$ and $V\left(\Gamma_{2}, \mathbf{G}_{2}\right)$. Their orbifold fundamental groups are isomorphic since $\Pi_{1}\left(\Gamma_{1}, \mathbf{G}_{1}\right) \simeq \Pi_{1}\left(\Gamma_{2}, \mathbf{G}_{2}\right)$ and we identify each of these groups with a group $E$. There is an extension

$$
1 \rightarrow F_{18} \rightarrow E \rightarrow D_{15} \rightarrow 1
$$

which determines (as in Theorem 1.3) two $D_{15}$-actions $\phi_{1}$ and $\phi_{2}$ on $V_{18}$ with $V_{18} / \phi_{1} \sim V\left(\Gamma_{1}, \mathbf{G}_{1}\right)$ and $V_{18} / \phi_{2} \sim V\left(\Gamma_{2}, \mathbf{G}_{2}\right)$. By construction, $\phi_{1}$ and $\phi_{2}$ must be homotopic, but they do not have the same quotient type since $V\left(\Gamma_{1}, \mathbf{G}_{1}\right)$ is not homeomorphic to $V\left(\Gamma_{2}, \mathbf{G}_{2}\right)$ (the "singular set" graphs of groups $\left(\Gamma_{1}, \mathbf{G}_{2}\right)$ and $\left(\Gamma_{2}, \mathbf{G}_{2}\right)$ are not isomorphic).

If $\mathbf{v}=(r, s, t)$ then recall the presentation for $\Pi_{1}^{\text {orb }}(\Gamma(\mathbf{v}), \mathbf{G}(\mathbf{v}))$ described in the beginning of $\S 2$. As in $\S 3$, we will abbreviate this group by $E(\Gamma(\mathbf{v}), \mathbf{G}(\mathbf{v}))$. If $C$ is a homotopy class of $\mathbf{Z}_{p}$-actions on $V_{g}(g>1)$ then there is an associated vector $\mathbf{v}$ so that $V_{g} / \phi \sim V(\Gamma(\mathbf{v}), \mathbf{G}(\mathbf{v}))$ for all $\phi \in C$ (Lemma 4.1).

In the remainder of this section we will use the following notation: if $\mathbf{n}$ is an element of $\left(\mathbf{Z}_{p}^{*}\right)^{J}$, then $\{\mathbf{n}\}$ will be the corresponding element of $\left(\mathbf{Z}_{p}^{*} /\langle 1\rangle\right)^{J}$ under the projection map. Moreover, by identifying $\left(\mathbf{Z}_{p}^{*} /\langle-1\rangle\right)$ with $\mathbf{Z}_{(p-1) / 2}$ we will view $\{\mathbf{n}\}$ as a vector in $\left(\mathbf{Z}_{(p-1) / 2}\right)^{J}$.

THEOREM 4.2. Let $p$ be a prime number and let $g>1$. In a homotopy class $C$ of $\mathbf{Z}_{p}$-actions on $V_{g}$ associated with $\mathbf{v}=(r, s, t)$, the set of strong equivalence classes $\mathscr{S} \mathscr{E}(C)$ is in 1-1 correspondence with $\left(\mathbf{Z}_{p}^{*}\right)^{s} \times\left(\mathbf{Z}_{p}^{*} /\langle-1\rangle\right)^{t}$. The correspondence depends on a choice of an exact sequence

$$
\varepsilon_{0}: 1 \rightarrow F_{g} \rightarrow \Pi_{1}^{\text {orb }}(V(\Gamma(\mathbf{v}), \mathbf{G}(\mathbf{v}))) \stackrel{\lambda_{0}}{\rightarrow} \mathbf{Z}_{p} \rightarrow 1 .
$$

If $\phi$ is a $\mathbf{Z}_{p}$-action whose strong equivalence class corresponds to $(\mathbf{m},\{\mathbf{n}\}) \in\left(\mathbf{Z}_{p}^{*}\right)^{s} \times$ $\left(\mathbf{Z}_{p}^{*} /\langle-1\rangle\right)^{t}$ then the equivalence class of $\phi$ corresponds to

$$
\left(\mathbf{v},\left[\left(\lambda_{0}\left(b_{j}\right) / m_{j}\right)_{j}\right],\left[\left(\lambda_{0}\left(d_{k}\right) / n_{k}\right)_{k}\right]\right) \in \mathscr{D}\left(\mathbf{Z}_{p}, V_{g}\right) .
$$

Proof. For each $(\mathbf{m}, \mathbf{n}) \in\left(\mathbf{Z}_{p}^{*}\right)^{s} \times\left(\mathbf{Z}_{p}^{*}\right)^{t}$ define

$$
\beta_{(\mathbf{m}, \mathbf{n})} \in \operatorname{Aut}(E(\Gamma(\mathbf{v}), \mathbf{G}(\mathbf{v})))
$$

by

$$
\beta_{(\mathbf{m}, \mathbf{n})}\left(a_{i}\right)=a_{i}, \quad \beta_{(\mathbf{m}, \mathbf{n})}\left(b_{j}\right)=b_{j}^{m_{j}}, \quad \beta_{(\mathbf{m}, \mathbf{n})}\left(c_{j}\right)=c_{j}, \quad \beta_{(\mathbf{m}, \mathbf{n})}\left(d_{k}\right)=d_{k}^{n_{k}} .
$$

Let $\beta \in \operatorname{Aut}(E(\Gamma(\mathbf{v}), \mathbf{G}(\mathbf{v})))$. As shown in the proof of Proposition 2.1, we may write $\beta$ as $\beta_{1} \beta_{2}$, where $\beta_{1} \in R$ and $\beta_{2}=\Pi \phi_{l}$. For each $i, j, k$ we have

$$
\beta_{2}\left(a_{i}\right)=a_{i}, \quad \beta_{2}\left(b_{j}\right)=b_{j}^{q_{j}}, \quad \beta_{2}\left(c_{j}\right)=b_{j}^{l_{j}} c_{j}^{\varepsilon_{j}}, \quad \beta_{2}\left(d_{k}\right)=d_{k}^{n_{k}}
$$


for some $q_{j}, l_{j}, n_{k} \in \mathbf{Z}_{p}^{*}$ and $\varepsilon_{j} \in\{ \pm 1\}$. Now define $\beta_{3} \in \operatorname{Aut}(E(\Gamma(\mathbf{v}), \mathbf{G}(\mathbf{v})))$ by

$$
\beta_{3}\left(a_{i}\right)=a_{i}, \quad \beta_{3}\left(b_{j}\right)=b_{j}^{\varepsilon_{j}}, \quad \beta_{3}\left(c_{j}\right)=b_{j}^{-l_{j}} c_{j}^{\varepsilon_{j}}, \quad \beta_{3}\left(d_{k}\right)=d_{k} .
$$

By Proposition 2.1, $\beta_{3} \in R$. Observe that $\beta_{3} \beta_{2}=\beta_{(\mathbf{m}, \mathbf{n})}$ where $m_{j}=\varepsilon_{j} q_{j}$. Therefore

$$
R \beta=R \beta_{1} \beta_{3}^{-1} \beta_{(\mathbf{m}, \mathbf{n})}=R \beta_{(\mathbf{m}, \mathbf{n})} .
$$

This shows that $R \backslash \operatorname{Aut}(E(\Gamma(\mathbf{v}), \mathbf{G}(\mathbf{v})))=\left\{R \beta_{(\mathbf{m}, \mathbf{n})} \mid(\mathbf{m}, \mathbf{n}) \in\left(\mathbf{Z}_{p}^{*}\right)^{s} \times\left(\mathbf{Z}_{p}^{*}\right)^{t}\right\}$.

A simple calculation using Proposition 2.1 now shows that $R \beta_{(\mathbf{m}, \mathbf{n})}=R \beta_{\left(\mathbf{m}^{\prime}, \mathbf{n}^{\prime}\right)}$ if and only if $\mathbf{m}^{\prime}=\mathbf{m}$ and each $n_{k}^{\prime}= \pm n_{k}$. This implies that $\left(\mathbf{Z}_{p}^{*}\right)^{s} \times\left(\mathbf{Z}_{p}^{*} /\langle-1\rangle\right)^{t}$ is in 1-1 correspondence with $R \backslash \operatorname{Aut}(E(\Gamma(\mathbf{v}), \mathbf{G}(\mathbf{v})))$. The first statement of the theorem now follows from Theorem 3.3.

If $\varepsilon_{0}$ is given and $\phi$ is a $\mathbf{Z}_{p}$-action whose strong equivalence class corresponds to $(\mathbf{m},\{\mathbf{n}\}) \in\left(\mathbf{Z}_{p}^{*}\right)^{s} \times\left(\mathbf{Z}_{p}^{*} /\langle-1\rangle\right)^{t}$, then, by Theorem 3.3, the equivalence class of $\phi$ corresponds to $\left[(\Gamma(\mathbf{v}), \mathbf{G}(\mathbf{v})), \lambda_{0} \beta_{(\mathbf{m}, \mathbf{n})}^{-1}\right] \in \Delta\left(\mathbf{Z}_{p}, V_{g}\right)$. Now recalling the description of $\Lambda$ given in the proof of Theorem 2.3, it can be seen that

$$
\Lambda^{-1}\left(\left[(\Gamma(\mathbf{v}), \mathbf{G}(\mathbf{v})), \lambda_{0} \beta_{(\mathbf{m}, \mathbf{n})}^{-1}\right]\right)=\left(\mathbf{v},\left[\left(\lambda_{0}\left(b_{j}\right) / m_{j}\right)_{j}\right],\left[\left(\lambda_{0}\left(d_{k}\right) / n_{k}\right)_{k}\right]\right)
$$

(note that the orbifold homeomorphism $F$ can be taken to be the identity map).

COROLlaRY 4.3. Consider the equivalence class of $\mathbf{Z}_{p}$-actions on $V_{g}(g>1)$ corresponding to $(\mathbf{v},[\mathbf{x}],[\mathbf{y}]) \in \mathscr{D}\left(\mathbf{Z}_{p}, V_{g}\right)$. The number of strong equivalence classes in the intersection of this equivalence class with each homotopy class associated with $\mathbf{v}$ is

$$
2^{s} s ! t ! / \prod_{i=1}^{(p-1) / 2}\left(a_{i} ! b_{i} !\right),
$$

where $a_{i}$ is the number of $i$ 's in $\{\mathbf{x}\}$ and $b_{i}$ is the number of $i$ 's in $\{\mathbf{y}\}$ for $1 \leq i \leq$ $(p-1) / 2$ (here we are using the notation described before Theorem 4.2).

ProOF. Let $\lambda_{0}: E(\Gamma(\mathbf{v}), \mathbf{G}(\mathbf{v})) \rightarrow \mathbf{Z}_{p}$ be the finite-injective epimorphism defined by $\lambda_{0}\left(a_{i}\right)=\lambda_{0}\left(b_{j}\right)=\lambda_{0}\left(c_{j}\right)=\lambda_{0}\left(d_{k}\right)=1$. This determines an exact sequence $\varepsilon_{0}$ as used in Theorem 4.2. Applying that theorem, we see that the set of strong equivalence classes in the intersection of the equivalence class corresponding to $(\mathbf{v},[\mathbf{x}],[\mathbf{y}])$ with a homotopy class associated with $\mathbf{v}$ is in $1-1$ correspondence with the set of pairs $(\mathbf{m},\{\mathbf{n}\}) \in\left(\mathbf{Z}_{p}^{*}\right)^{s} \times\left(\mathbf{Z}_{p}^{*} /\langle-1\rangle\right)^{t}$ which satisfy $\left[\left(1 / m_{j}\right)_{j}\right]=[\mathbf{x}]$ and $\left[\left(1 / n_{k}\right)_{k}\right]=[\mathbf{y}]$. Then the number of all possible $\mathbf{m}$ 's in $\left(\mathbf{Z}_{p}^{*}\right)^{s}$ is $2^{s}$ times the order of the orbit of $\left(1 / m_{j}\right)_{j}$ under the $\sum_{s}$ action on $\left(\mathbf{Z}_{p}^{*}\right)^{s}$ and this equals $2^{s} s ! / \prod_{i=1}^{(p-1) / 2} a_{i}$ !. Similarly the number of possible $n$ 's is $t ! / \prod_{i=1}^{(p-1) / 2} b_{i}$ ! and the corollary follows.

COROLLARY 4.4: Let $g>1$.

(a) Involutions on $V_{g}$ which are homotopic are strongly equivalent.

(b) Homotopic $\mathbf{Z}_{3}$-actions on $V_{g}$ are equivalent but need not be strongly equivalent unless $g=2$. When $g=2$ homotopic $\mathbf{Z}_{3}$-actions are strongly equivalent. Homotopic $\mathbf{Z}_{3}$-actions which do not have a circle component in their fixed point sets are strongly equivalent. 
(c) For prime $p>3$, homotopic $\mathbf{Z}_{p}$-actions on $V_{g}$ need not be equivalent, and actions which are both homotopic and equivalent need not be strongly equivalent. Homotopic free $\mathbf{Z}_{p}$-actions are strongly equivalent.

PROOF. Part (a) follows immediately from Theorem 4.2 since, when $p=2$, $\left|\mathbf{Z}_{p}^{*}\right|=1$.

Now consider the homotopy class of a $\mathbf{Z}_{p}$-action $\phi$ on $V_{g}$ which is associated with $\mathbf{v}=(r, s, t)$ where $g=p(r+s)+(p-1)(t-1)$. By Proposition 2.6 the number of equivalence classes in this homotopy class is equal to the number of equivalence classes in the set of $\mathbf{Z}_{p}$-actions associated with $\mathbf{v}$. This latter number in turn equals

$$
N_{s} N_{t}=\left(\begin{array}{c}
s+(p-3) / 2 \\
(p-3) / 2
\end{array}\right)\left(\begin{array}{c}
t+(p-3) / 2 \\
(p-3) / 2
\end{array}\right)
$$

as shown in the proof of Corollary 2.4. If $p=3$ then $N_{s} N_{t}=1$, so any $\mathbf{Z}_{3}$-action which is homotopic to $\phi$ is equivalent to $\phi$. If $\phi$ is a free action, then $s=t=0$ and $N_{s} N_{t}=1$; thus homotopic free $\mathbf{Z}_{p}$-actions are equivalent. Otherwise, if $p>3$ and $s+t>0$ then $N_{s} N_{t}>1$; so homotopic $\mathbf{Z}_{p}$-actions need not be equivalent.

Next consider the set of $\mathbf{Z}_{p}$-actions which are both homotopic and equivalent to $\phi$. Let $N$ be the number of strong equivalence classes in this set; thus $N$ is given by Corollary 4.3. If $\phi$ is a free action then $N=1$. It follows (using one of the results of the previous paragraph) that homotopic free $\mathbf{Z}_{p}$-actions are strongly equivalent as claimed in (c). If $p=3$ then $\left|\mathbf{Z}_{p}^{*} /\langle-1\rangle\right|=1$ and $N=2^{s}$. In this case, if $s=0$ (so that $\phi$ has no circle component in its fixed point set), then $N=1$ and a $\mathbf{Z}_{3}$-action which is homotopic to $\phi$ must be strongly equivalent to $\phi$. If $p=3$ and $g=2$, then $3(r+s)+2(t-1)=2$ so that $r+s=0$, and again any action homotopic to $\phi$ must be strongly equivalent to $\phi$. Finally, if $p=3$ and $g>2$, or if $p>3$ then there will be choices for $\mathbf{x}$ and $\mathbf{y}$ so that if $\phi$ corresponds to $(\mathbf{v},[\mathbf{x}],[\mathbf{y}])$ then $N>1$, showing that, in these cases, $\mathbf{Z}_{p}$-actions which are homotopic and equivalent need not be strongly equivalent.

\section{REFERENCES}

[E1] A. Edmonds, Transformation groups and low-dimensional manifolds, Group Actions on Manifolds, Contemp. Math., vol. 36, Amer. Math. Soc., Providence, R.I., 1985.

[E2] _ - On the equivariant Dehn lemma, Combinatorial Methods in Topology and Algebraic Geometry, Contemp. Math., vol. 44, Amer. Math. Soc., Providence, R.I., 1985.

[E3] _ Surface symmetry. I, Michigan Math. J. 30 (1982), 171-183.

[F-R] D. I. Fuchs-Rabinovitch, On the automorphism group of free products. I, Mat. Sb. 8 (1940), 265-276.

[G] K. W. Gruenberg, Combinatorial topics in group theory, Lecture Notes in Math., vol. 143, Springer-Verlag, New York, 1970.

[K] S. Kerckhoff, The Nielsen realization problem, Ann. of Math. (2) 117 (1983), 235-265.

[M] A. Marden, Isomorphisms between Fuchsian groups, Advances in Complex Function Theory, Lecture Notes in Math., vol. 505, Springer-Verlag, New York.

[MB] J. Morgan and H. Bass (ed.), The Smith conjecture, Academic Press, Orlando, Fla., 1984.

[MM] D. McCullough and A. Miller, Homeomorphisms of 3-manifolds with compressible boundary, Mem. Amer. Math. Soc., No. 344 (1986).

[MMZ] D. McCullough, A. Miller, and B. Zimmermann, Group actions on handlebodies, preprint.

[MY] Wm. Meeks and S-T. Yau, The equivariant Dehn's lemma and loop theorem, Comment. Math. Helv. 56 (1981), 225239.

[P] J. Przytycki, Free actions of $\mathbf{Z}_{n}$ on handlebodies and surfaces, Bull. Acad. Polon. Sci. 26 (1978), 617-624. 
[S] P. A. Smith, Abelian actions on 2-manifolds, Michigan Math. J. 14 (1967), 257-275.

[T] Wm. Thurston, Three-manifolds with symmetry, preprint, 1982.

[ZZ] H. Zieschang and B. Zimmermann, Endliche gruppen von abbildungsklassen gefaserter 3-mannigfaltigkeiten, Math. Ann. 240 (1979), 41-62.

[Z] B. Zimmermann, Über Abbildungsklassen von Henkelkörpern, Arch. Math. 3 (1979), 379-382.

Department of Mathematics, Saint Louis University, St. Louis, Missouri 63103

Department of MAThematics, UNiVERSity of OKLAHOMA, NoRMAN, OKLAHOMA 73019 